\title{
Using a coarse-grained modeling framework to identify oligomeric motifs with tunable secondary structure
}

\author{
Christopher C. Walker, ${ }^{\dagger, \ddagger}$ Garrett A. Meek, ${ }^{\dagger, \ddagger}$ Theodore L. Fobe, ${ }^{\dagger}$ and Michael R. \\ Shirts ${ }^{*, \dagger}$ \\ $\dagger$ Department of Chemical and Biological Engineering \\ University of Colorado Boulder, Boulder, CO \\ $\ddagger$ These authors contributed equally to this work \\ E-mail: michael.shirts@colorado.edu
}

\begin{abstract}
Coarse-grained modeling can be used to explore general theories that are independent of specific chemical detail. In this paper, we present cg_openmm, a Python-based simulation framework for modeling coarse-grained hetero-oligomers and screening them for structural and thermodynamic characteristics of cooperative secondary structures. cg_openmm facilitates the building of coarse-grained topology and random starting configurations, setup of GPU-accelerated replica exchange molecular dynamics (REMD) simulations with the OpenMM software package, and features a suite of post-processing thermodynamic and structural analysis tools. In particular, native contacts analysis, heat capacity calculations, and free energy of folding calculations are used to identify and characterize cooperative folding transitions and stable secondary structures. In this work, we demonstrate the capabilities of cg_openmm on a simple 1-1 LennardJones coarse-grained model, in which each residue contains 1 backbone and 1 sidechain
\end{abstract}


bead. By scanning both non-bonded and bonded force field parameter space at the coarse-grained level, we identify and characterize sets of parameters which result in the formation of stable helices through cooperative folding transitions. Moreover, we show that the geometries and stabilities of these helices can be tuned by manipulating the force field parameters.

\section{Introduction}

Foldamers, oligomeric molecules which form secondary structure in solution due to noncovalent interactions amongst nonadjacent residues, show great promise for a multitude of applications including therapeutics, ${ }^{1}$ antimicrobials,${ }^{2,3}$ catalysis, ${ }^{4,5}$ molecular sensors,${ }^{6}$ and nanostructured materials. ${ }^{7,8}$ In the past few decades, many research studies have elucidated and characterized new backbone and sidechain chemistries leading to secondary structure formation. ${ }^{9-19}$ In order to design foldamers exhibiting stable, tunable tertiary structures, which remains a formidable challenge but a necessary step in achieving the structural specificity required in many applications, it is important to first gain a solid understanding of the design principles for secondary structure formation.

Due to the challenges associated with the synthesis and especially the characterization of potential foldamer molecules, molecular dynamics (MD) simulations are a vital tool for searching broad chemical spaces and efficiently exploring folding properties such as stability and cooperativity. Moreover, MD simulations provide a direct atomic-level (or coarsegrained particle-level) view of secondary structures. Recent progress in enhanced sampling methods, ${ }^{20,21}$ statistical analysis techniques, ${ }^{22,23}$ sheer computing power, and the quality of both atomistic and coarse-grained force fields have paved the way for increasingly accurate simulations of molecular folding. ${ }^{24}$

Previous experimental and simulation efforts of foldamers have largely focused on biosimilar molecules resembling proteins, DNA, or RNA. Yet, biological foldamers represent only a 
miniscule fraction of chemical space, and their chemical sequences have been constrained by evolutionary pressure to perform specific biological functions in a specific biological environment. Without these constraints, carefully designed foldamers with non-natural backbones can have several advantages - for example, they can be more chemically resilient, especially with regard to resistance to hydrolysis, or exhibit superior mechanical and electrical properties not possible with a peptidic backbone.

Knowledge-based approaches to modeling proteins and biosimilar molecules, such as Rosetta, ${ }^{25}$ have benefited greatly from a wealth of experimental crystal structure data available in the PDB databank ${ }^{26}$ and elsewhere. The scarcity of experimental data of any kind for non-biological foldamers, in contrast, has hindered the development of new chemically specific non-biological foldamer models. Reasons for this lack of data include the generally tedious nature of synthesizing sequence-defined hetero-oligomers, and that techniques for characterizing the sequences of non-biological hetero-oligomers lag behind those from biological hetero-oligomers (proteomics, genomics, etc.). ${ }^{27}$

Many biologically important foldamers, as a key part of their function, are also marginally stable. Achieving the correct balance between entropy and enthalpy is frequently beyond the limits of accuracy for classical atomistic force fields, requiring careful refinement of force field parameters and/or the consideration of more complex energetic terms such as atomic polarizabilities. ${ }^{28}$ Thus, rather than using bottom-up approaches based on quantum level simulations to develop an accurate atomistic force field for a specific foldamer system, we take a different approach, choosing to develop a coarse-grained simulation framework for studying non-chemically specific behaviors of categories of foldamers.

Coarse-grained molecular models, in which atoms are grouped together into effective interaction sites, allow for larger time and length scales to be accessed, and the reduced degrees of freedom compared to all-atom models lead to a smoother energy landscape. Numerous studies spanning more than four decades have investigated different ways of parameterizing coarse-grained models intended to predict or reproduce the secondary structure 
of biomolecules. ${ }^{29,30}$ Most coarse-grained models of proteins or foldamers are either 1) inherently biased towards specific targeted structures (such as Gō-type models and elastic network models), or 2) parameterized to chemically specific atomistic simulation and/or experimental data. In Gō-type coarse-grained protein models, nonbonded interactions are restricted to native contact pairs, artificially stabilizing secondary structure. Nonetheless, Gō-type structure-based models have contributed valuable insight into protein folding mechanisms. ${ }^{30,31}$ Chemically specific coarse-grained models are typically parameterized using systematic coarse-graining techniques including iterative Boltzmann inversion, ${ }^{32}$ forcematching, ${ }^{33}$ relative entropy methods, ${ }^{34}$ or more recently, by machine learning techniques. ${ }^{35}$

This work is intended to improve understanding of foldamer behavior at a more fundamental level through the use of generic coarse-grained models free of chemical specificity. A reduced force field parameter space allows for efficient parameter scanning and optimization when searching for foldamers with novel characteristics. Coarse-grained modeling also enables efficient investigation of overall forces driving oligomer secondary structure formation, such as stiffness of the backbone and sidechains, interaction strengths between backbone and sidechains, and size of the sidechains.

In this work, we introduce cg_openmm, a Python-based framework for setup, execution, and thermodynamic analysis of coarse-grained foldamer replica exchange molecular dynamics (REMD) simulations, and analyze_foldamers, a companion Python package for structural analysis of coarse-grained foldamers. These software packages serve as the foundation of a broader effort to understand heteropolymer secondary and tertiary structure formation as a function of molecular parameters.

To demonstrate the utility of these tools for exploring the structural and thermodynamic properties of oligomers that exhibit secondary structure, we use an illustrative example of a helix-forming Lennard-Jones homo-oligomer containing 1 backbone bead and 1 sidechain bead per residue throughout this work. Using initial estimates of nonbonded (Lennard Jones 12-6) and bonded (bond stretching, bond-bending, and periodic torsion) parameters derived 
from a pre-designed helix structure, we systematically vary sets of force field parameters and analyze their effects on helix stability, geometry, and cooperativity of folding. Additionally, we investigate the effect of chain length on these properties.

In the subsequent section, we discuss our coarse-grained homo-oligomer model and the setup of REMD simulations. Then we describe in detail the capabilities of our thermodynamic analysis tools, including heat capacity and free energy of folding calculations, and structural analysis tools, including bonded parameter distributions and native structure determination from structure-based clustering. Next we present the results of scanning the sensitivity of helix stability and cooperativity on sets of bonded and nonbonded parameters. Finally, we discuss future work enabled by the cg_openmm framework, including the potential for discovering novel non-canonical secondary structures, designing stimuli-responsive foldamer systems which transition between multiple stable secondary structures, and gaining a deeper understanding of the principles governing folding through rapid hypothesis testing.

\section{Methods}

\subsection{Coarse-grained model}




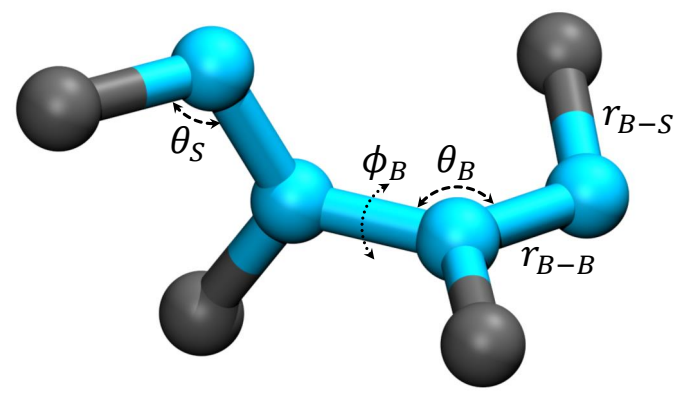

Figure 1: Illustration of the topology and bonded parameters of a 1-backbone, 1-sidechain (1-1) homo-oligomer containing 4 residues.

Figure 1 depicts the topology of the simple coarse-grained homo-oligomer model used throughout this work, containing one backbone bead (blue) and one sidechain bead (black) per residue. Our model draws inspiration from that of Tozzini ${ }^{36}$ in which the standard $\phi$ and $\psi$ torsional angles of atomistic oligopeptides were mapped to effective coarse-grained angles and pseudotorsions. In our model, bond distances between backbone-backbone and backbone-sidechain beads are labeled as $r_{B-B}$ and $r_{B-S}$, respectively. $\theta_{B}$ is the effective bond-bending angle defined by three consecutive backbone beads and $\theta_{S}$ is the effective bondbending angle of two backbone beads and a sidechain bead. $\phi_{B}$ is the backbone pseudotorsion angle defined by the positions of four consecutive backbone beads. Other pseudotorsion angles involving sidechain beads are not depicted in Figure 1 for simplicity.

The bonded potential functional forms used throughout this work are given in eqs 1-3. Bond-stretching and bond angle-bending potentials are harmonic functions, and torsions are given by a periodic function, in which $n$ is periodicity, $k_{t}$ is the force constant, and $\phi_{t}$ is the phase shift. Torsions with a periodicity of 3 represent the familiar rotational energy landscape with 3 minima, typically for gauche+, gauche-, and trans states. A periodicity of 1 corresponds to a single minimum, with $\phi_{t}$ being strongly disfavored. Such a potential applied to the backbone beads can favor the formation of helices. ${ }^{37}$ At the atomistic level, such asymmetric pseudotorsion potentials could arise from steric interactions amongst neighboring residues. In this work, only periodic torsions with $n=1$ are considered. While we have 
chosen these relatively simple potentials in this initial study, nearly arbitrary custom bonded potential functions may be specified in OpenMM. ${ }^{38}$

$$
\begin{gathered}
U_{\text {bond }}=k_{b}\left(r-r_{o}\right)^{2} \\
U_{\text {angle }}=k_{a}\left(\theta-\theta_{o}\right)^{2} \\
U_{\text {torsion }}=k_{t}\left[1+\cos \left(n \phi-\phi_{t}\right)\right]
\end{gathered}
$$

Nonbonded interactions are described by a Lennard-Jones 12-6 function with no cutoff, due to the small size of the foldamer systems studied in this work.

$$
U_{\text {nonbond }}=4 \varepsilon\left[\left(\frac{\sigma}{r}\right)^{12}-\left(\frac{\sigma}{r}\right)^{6}\right]
$$

Nonbonded interactions for 1-2 and 1-3 bonded neighbors are excluded, and 1-4 interactions are given full weight. Nonbonded interactions between unlike particles are described by standard Lorentz-Berthelot mixing rules:

$$
\begin{gathered}
\sigma_{i j}=\frac{\sigma_{i i}+\sigma_{j j}}{2} \\
\varepsilon_{i j}=\sqrt{\varepsilon_{i i} \varepsilon_{j j}}
\end{gathered}
$$

Highly customized nonbonded potentials can be defined in OpenMM, allowing for future studies of complex foldamer systems. Coulombic interactions are not explicitly considered here at this time, as we are using a coarse-graining philosophy where Coulombic interactions can usually be treated as averaged out for neutral molecules. ${ }^{39}$

Throughout this work, reduced units for distance and energy are used: the base unit for length is the sigma parameter of the backbone bead $\left(\sigma_{B}\right)$, and the base unit for energy is the epsilon parameter of the backbone bead $\left(\varepsilon_{B}\right)$. 


\subsection{Overview of cg_openmm}

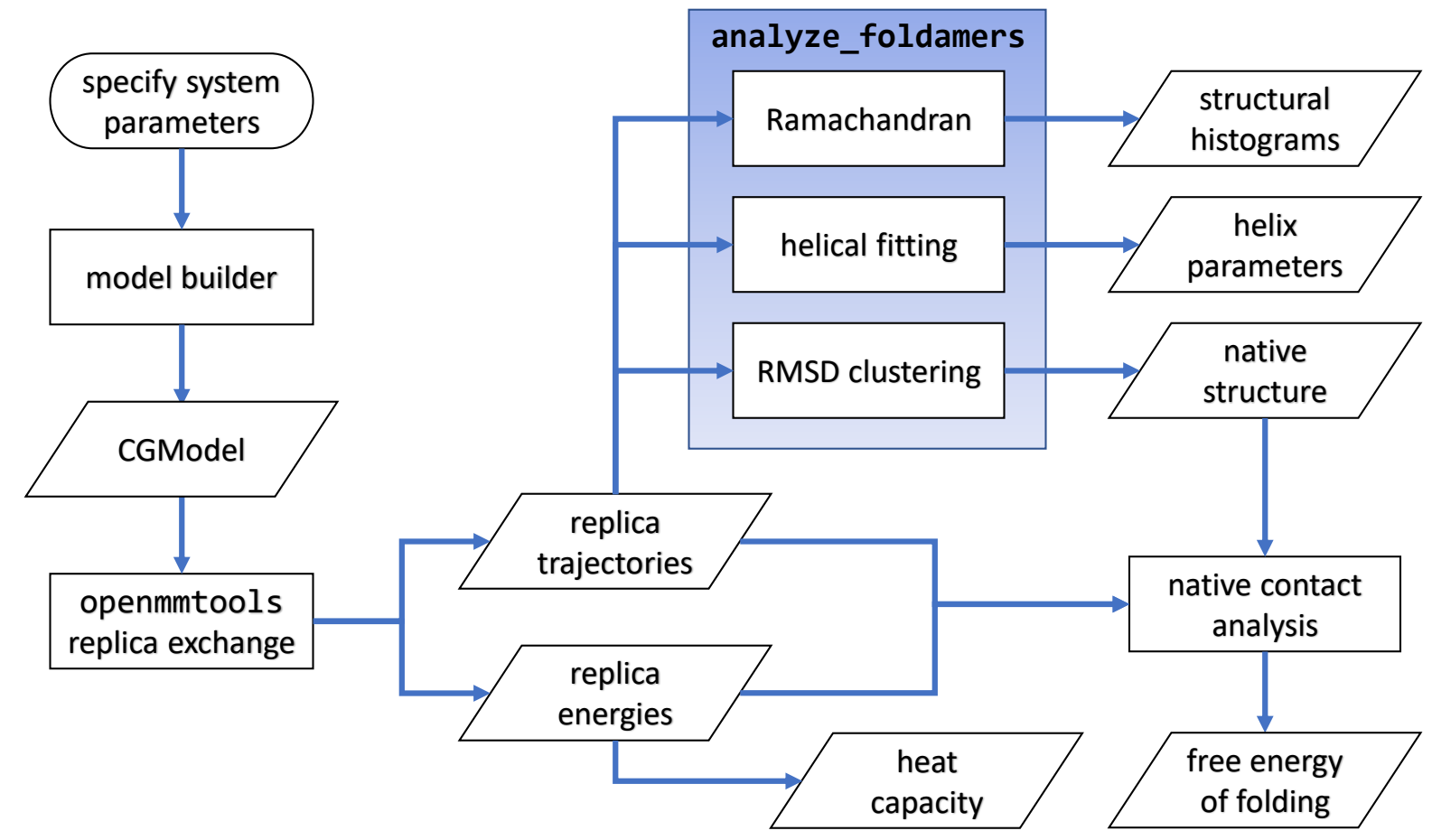

Figure 2: Illustration of the flow of data within different modules of cg_openmm and analyze_foldamers. Input parameters are represented as a rounded rectangle, processes as rectangles, and data as parallelograms. Structural analysis is performed within analyze_foldamers, as indicated by processes in the blue shaded region.

The flow of data in the cg_openmm and analyze_foldamers Python packages is illustrated in Figure 2. First, the user specifies parameters, or ranges of parameters to scan, of the oligomer system. These include particle definitions, monomer topologies, oligomer length and residue sequence, bonded force field parameters, and nonbonded force field parameters. Initial particle coordinates may be either supplied from an existing file, or generated using a self-avoiding random walk algorithm. All of the above information about an oligomer system is stored in a CGModel class object. When initially constructed, the CGModel class generates an OpenMM topology object, which contains the model's bond, angle, and torsion definitions, and an OpenMM system object, which contains the model's force field parame- 
ters. The CGModel, in combination with the energy and trajectory coordinate files generated by OpenMM, is used to perform subsequent thermodynamic and structural analyses.

\subsection{Replica Exchange Molecular Dynamics Simulations}

To overcome the slow sampling at thermodynamic states near or below the folding transition temperature, a common strategy in foldamer and protein simulation literature is to perform replica exchange molecular dynamics (REMD) simulations. ${ }^{40-43}$ In REMD, simulations are run concurrently in separate simulation boxes, with configurational swaps between boxes attempted periodically. Typically, the parallel simulation cells differ in temperature (parallel tempering). These swaps are either accepted, in which the configurations of the two simulation boxes are switched, or rejected, in which the configurations stay the same, in accordance with a Metropolis acceptance criterion:

$$
P_{\text {accept }}\left(i, x_{i}, j, x_{j}\right)=\min \left\{1, \frac{\exp \left[\beta_{i} U_{i}\left(x_{j}\right)+\beta_{j} U_{j}\left(x_{i}\right)\right]}{\exp \left[\beta_{i} U_{i}\left(x_{i}\right)+\beta_{j} U_{j}\left(x_{j}\right)\right]}\right\}
$$

In eq 7 , the subscripts denote state index, $x$ represents the configuration, $U$ is potential energy, and $\beta=1 / k_{B} T$. Upon an accepted swap the system can escape metastable states at local energy minima, and more effectively navigate to the global energy minimum.

The efficiency of replica exchange simulations depends greatly on the temperature states used, as well as the frequency at which exchange attempts are made. ${ }^{44}$ It is now generally accepted that exchange attempts should be made as often as possible ${ }^{45}$ but this should be balanced with the computational overhead of the exchange moves. Logarithmically spaced replica temperatures have been shown to provide roughly equal acceptance ratios in parallel tempering simulations of systems which have constant heat capacity. ${ }^{46}$ However, in systems which undergo structural changes with a significant free energy barrier (such as folding/unfolding), a bottleneck for the migration of replicas can occur near the transition 
when logarithmic spacing is used. ${ }^{47} \mathrm{~A}$ number of solutions for improving sampling near the folding transition have been proposed. ${ }^{47-49}$ One strategy, which we have adopted in this work, is to optimize the spacing of temperatures such that the entropy change between all adjacent states is equal. ${ }^{48}$ If heat capacity data as a function of temperature is available (i.e., estimated from a preliminary simulation with logarithmic spacing), temperatures can be selected such that all adjacent pairs $T_{i}$ and $T_{i+1}$ satisfy the following integral equation:

$$
\int_{T_{i}}^{T_{i+1}} \frac{C_{V}(T)}{T} d T=\frac{\Delta S}{N}
$$

In eq $8, C_{V}(T)$ is the temperature-dependent constant volume heat capacity, $N$ is the total number of replicas, and $\Delta S$ is the constant entropy increase (CEI) across all states. Key metrics for assessing the sampling efficiency of replica exchange simulations include the number of transits between the lowest and highest temperatures over a given time period, and the transition matrix of acceptance probabilities. ${ }^{44,50,51}$

The replica exchange simulations in cg_openmm are executed using the GPU-accelerated openmmtools multistate framework ${ }^{51-54}$ in the canonical ensemble. Even for the small systems considered here, we have found that GPU acceleration provides a dramatic speedup compared to running in parallel with $1 \mathrm{CPU} /$ replica. In this work, 12 replicas were used in all cases, spanning a reduced temperature range of $200 K\left(k_{B} / \varepsilon_{B}\right)$ to $600 K\left(k_{B} / \varepsilon_{B}\right)$. A Langevin integrator with a collision frequency of $5 \mathrm{ps}^{-1}$ and timestep of $5 \mathrm{fs}$ was used. We chose this slightly conservative time step to avoid instabilities that may arise when exploring wide ranges of force field parameters. Replica exchange moves were attempted between neighboring temperature pairs after every 200 timesteps (1 ps), which we found to provide a good balance between computational efficiency and replica sampling efficiency. It should be noted that openmmtools supports a variety of other integrators, including Metropolized variants, in which the molecular dynamics segments are themselves subject to acceptance or rejection. ${ }^{55}$ In a preliminary replica exchange simulation 50 ns in duration, logarithmic 
temperature spacing was used. We then applied the constant entropy increase method as outlined above to the preliminary heat capacity data to refine the temperature spacing for a 200 ns production REMD simulation. To solve for the constant entropy increase temperature spacings, the $\frac{C_{V}}{T}$ data was splined such that arbitrary definite integrals could be accurately evaluated. The temperature spacings were then optimized ${ }^{56}$ to minimize the standard deviation of the entropy increases, with the constraint that the temperature range be the same as the original. A comparison of replica exchange transition matrices from simulations with logarithmic temperature spacing and optimized CEI spacing is shown for an example system in the supporting information (Figure S1).

Unless otherwise stated, for both the preliminary $50 \mathrm{~ns}$ log-spaced simulation and the 200 ns CEI-spaced simulation, the first $20 \mathrm{~ns}$ is discarded as an equilibration period, and potential energy time series data for each thermodynamic state is decorrelated using the pyMBAR timeseries module. ${ }^{22}$ Running on one NVIDIA ${ }^{\circledR}$ Tesla ${ }^{\circledR}$ V100 GPU, these combined 250 ns of REMD simulations (totaling $3 \mu \mathrm{s}$ over all replicas) took approximately 8 hours to complete. In general, the energy decorrelation times for each thermodynamic state vary significantly, with states near the folding transition exhibiting slower decorrelation than lowtemperature folded states. To estimate an overall energy decorrelation time to apply to all states, we fit the individual decorrelation times to a normal distribution, and chose the time which yields a cumulative probability distribution value of $\left(2 n_{\text {replica }}-1\right) /\left(2 n_{\text {replica }}\right)$. For 12 replicas, this corresponds to 1.73 standard deviations above the mean decorrelation time. The decorrelation of replica exchange time series data remains an active topic of research and will be revisited in future work. We used the physical_validation Python package $^{57,58}$ to confirm that the replica exchange simulations sample from the canonical Boltzmann distribution (for further details, see supporting information, Section S2).

In the following sections, we describe the analysis tools included in cg_openmm and analyze_foldamers for screening thermodynamic and structural properties of foldamer candidates. 


\subsection{Heat capacity}

Heat capacity curves as a function of temperature are a simple probe for determining whether and where a folding transition takes place. The melting point of a foldamer appears as a peak in heat capacity because thermodynamic work is required to break/make the native non-covalent interactions. With a fixed size simulation box, constant volume heat capacity $\left(C_{V}\right)$ is computed from the derivative of decorrelated potential energy with respect to temperature using a finite difference scheme. The Multistate Bennett Acceptance Ratio (MBAR) method ${ }^{22}$ is used to compute the expectation value of heat capacity at temperatures of interest, including arbitrary intermediate temperatures not sampled during the replica exchange simulation. By reweighting the reduced potential energies from a replica exchange simulation to the temperatures of interest, the energies of each uncorrelated configuration sampled at each thermodynamic state can be leveraged in the computation of $C_{V}$ at all states of interest, provided that there is sufficient configurational overlap between the thermodynamic states. Moreover, MBAR provides an asymptotically unbiased estimate of uncertainty at both sampled and unsampled states. In this work we have utilized the MBAR implementation in the Python package pymbar. ${ }^{22}$

For all expectation calculations of thermodynamic observables, including heat capacity, native contact fraction, and free energy of folding as a function of $\mathrm{T}$, uncertainties can also be reliably calculated using bootstrap resampling. ${ }^{59}$ Uncertainty in detailed features of the heat capacity curve, such as melting point and full-width half maximum, can also be computed in this way. In this work, we implement a variant of standard bootstrapping in which the initial reference frame for slicing decorrelated time series data is also randomly selected in each bootstrap trial. This effectively allows all data points to be utilized in the calculation, leading to smoother mean values. 


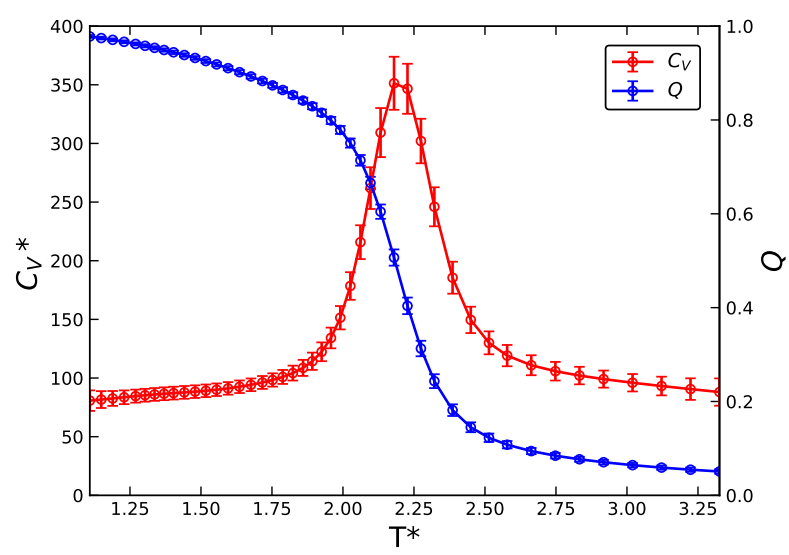

Figure 3: Example of a heat capacity $\left(C_{V}\right)$ expectation curve and a native contact fraction $(Q)$ expectation curve, with 3 unsampled states between each sampled state included in the MBAR calculation. All quantities are given in a reduced units system, in which $\varepsilon_{B}$ is the base energy unit. Uncertainties are estimated as the standard deviation over all bootstrap trials.

\subsection{Native state from clustering}

A challenging but critical aspect of studying novel foldamers is identifying the native configuration in the folded state(s), without any prior knowledge or expectation. Key parameters defining the native state are the specific pairs of particles which are in proximity to one another, and the distances separating these native pairs. The fraction of native contacts is an important metric for classifying each conformation in a simulation trajectory as 'folded' or 'unfolded', which is necessary for computing the free energy of folding and other related thermodynamic quantities.

One approach to estimating the native state is by clustering configurations from a replica exchange simulation. In general, clustering methods aim to optimize the placement of data points into groups (clusters) based on their similarity to one another. Applied to molecular simulation trajectory data, clustering methods can identify groups of similar structures representative of frequently visited conformations. ${ }^{60}$ The cluster with the most similar members is likely a candidate for the native structure, whereas other clusters may represent metastable 
states.

In analyze_foldamers, we have implemented clustering based on two different metrics: root-mean-square deviation (RMSD) of particle coordinates, and distance of the vector of backbone torsion angles. In the full coordinate RMSD approach, structures from all uncorrelated frames in the trajectory are first superimposed to an arbitrary reference frame using a rotational and translational optimization scheme. ${ }^{61}$ For each pair of frames, the coordinate RMSD distance matrix is the following:

$$
d_{R M S D}(i, j)=\sqrt{\frac{1}{n} \sum_{k=1}^{n}\left(\boldsymbol{x}_{i, k}-\boldsymbol{x}_{j, k}\right)^{2}}
$$

In which $i$ and $j$ are the frame indices, $x$ represents the Cartesian coordinates of particle $k$, and $n$ is the total number of particles in the molecule. In the torsion clustering approach, the distance matrix is composed of distances in the space of backbone torsion angles, between each pair of frames:

$$
d_{\phi}(i, j)=\sqrt{\sum_{k=1}^{N-2}\left(\phi_{i, k}-\phi_{j, k}\right)^{2}}
$$

In the above equation, $\phi_{i, k}$ is the $k^{t h}$ backbone torsion angle in frame $i$. In a chain with $N$ backbone particles, there are a total of $N-2$ backbone torsions. Note that periodic boundary conditions must be applied in each dimension.

In a typical trajectory set from REMD simulations of a potential foldamer, structures above the melting temperature will be characterized by large distances between data points, especially in the case of particle coordinate RMSD, and structures well below the melting temperature will be tightly distributed. Density-based clustering methods, which identify clusters of high density and discard low density regions as 'noise' points, are well suited to this application. In particular, we have chosen to use the Density Based Spatial Clustering of Applications with Noise (DBSCAN) $\operatorname{method}^{62,63}$ implemented in the scikit-learn python package. ${ }^{64}$ DBSCAN is highly efficient in that only one pass through the data is performed, 
and it does not require that the number of clusters be known a priori. This is in contrast to center-based methods such as K-means clustering, which aims to minimize the intra-cluster sum of squares against the cluster mean, or K-medoids, which aims to minimize the sum of distances between cluster member data and the medoid in each cluster. In general, the number of metastable states of a foldamer system is not known, making it necessary to repeat K-means or K-medoids clustering several times with different numbers of clusters to find an optimal number of clusters. DBSCAN uses two key parameters - the number of minimum samples $\left(n_{\min }\right)$ within the radius $(\epsilon)$ required to classify a data point as a 'core point'. Points that lie within $\epsilon$ of a core point, but do not satisfy the core point criteria, are non-core cluster members. Any point that lies beyond $\epsilon$ of a core point is classified as noise. One disadvantage to density-based clustering methods is that there is no direct analog to the 'centroid' or 'medoid' structures, as are returned by K-means and K-medoids, respectively. However, by constructing a similarity matrix $\left(S_{i j}\right)$ for each cluster, a representative structure of that cluster can be estimated:

$$
\begin{gathered}
S_{i j}=\exp \left(\frac{d_{i, j}}{d_{\text {scale }}}\right) \\
\text { medoid index }=\operatorname{argmax}\left(\sum_{j} S_{i j}\right)
\end{gathered}
$$

In eq $11, d_{\text {scale }}$ is an arbitrary scaling factor. To improve efficiency when dealing with large datasets containing primarily unfolded, "noisy" structures, we have implemented a preclustering filtering function, to remove data points from the distance matrix which have few near neighbors. The fraction of data passing through the density filter is specified by the user, and a neighborhood scan radius parameter and minimum number of neighbors within that radius are optimized to achieve the specified filtering criteria. As a result, only relatively high-density data points are input into the DBSCAN algorithm, allowing the use of a smaller epsilon parameter to identify distinct structures more precisely. A similar density filtering pre-clustering step has been successfully used to identify protein-ligand binding sites in a 
noisy trajectory dataset. ${ }^{65}$

\subsection{Native contact fraction calculation}

Once the native structure(s) are established, native contact pairs are identified as particles which are less than a distance cutoff $c_{c u t}$ from each other. Then, the pairwise distances between native contacts in the native structure $\left(r_{\text {native }}\right)$ are used to compute the fraction of native contacts $(Q)$ of each uncorrelated frame in the replica exchange trajectory set. A multiplicative tolerance factor $\left(c_{t o l}\right)$ is applied when scanning the trajectories for native contacts. Thus, a pair is considered a native contact if 1 ) it is a native pair defined in the native structure, and 2) the distance between two particles is less than or equal to $r_{n a t i v e} c_{t o l}$. Using MBAR, the expectation of $Q$ as a function of temperature is computed from the reduced energies evaluated at all thermodynamic states of interest and the corresponding native contact fractions. In a well-defined foldamer system which transitions from a disordered state to a single folded state, the $Q$ vs $T$ data is well-described by a sigmoidal hyperbolic fitting function:

$$
Q(T)=\frac{Q_{0}+Q_{1}}{2}-\frac{Q_{0}-Q_{1}}{2}\left[\tanh \left(\frac{T-T_{0}}{d}\right)\right]
$$

In which $Q_{0}$ is the native contact fraction at the low-temperature limit, $Q_{1}$ is the native contact fraction at the high-temperature limit, and $d$ is a sigmoid width parameter which describes the temperature range over which the folding transition occurs. The $d$ parameter is thus a measure of the cooperativity of the transition.

In general, $c_{c u t}$ and $c_{t o l}$ are varied in a $2 \mathrm{D}$ optimization scheme to maximize $Q_{1}-Q_{0}$ (i.e., maximize the distinction between the folded and unfolded states). A similar result may be achieved by minimizing sigmoid width $(d)$ parameter (i.e., maximize observed cooperativity). 


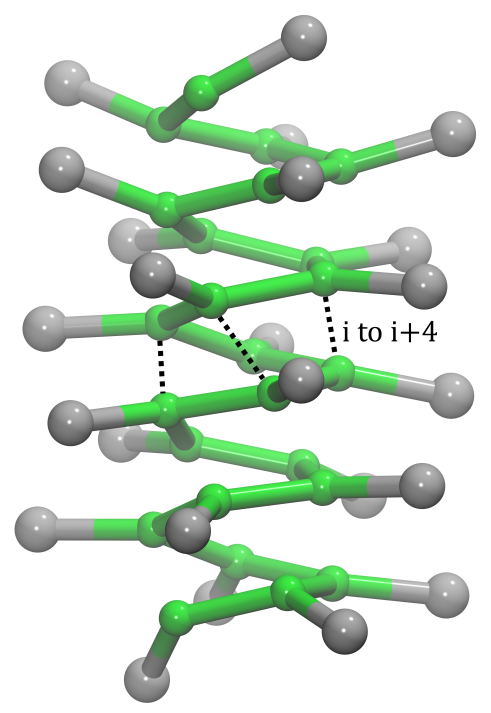

Figure 4: Illustration of native contact pairs (indicated by dashed lines) along the helical backbone for an $i$ to $i+4$ helical foldamer.

For helices, as is the case for all examples considered in this work, we have also implemented a simplified native contacts optimization scheme in cg_openmm. The average distances between all backbone-backbone bead pairs in the nonbonded interaction list are computed as a function of the number of bonds separating them $(i$ to $i+3, i$ to $i+4$, etc.), and native contacts are chosen to be the set of pairs $(i$ to $i+n)$ exhibiting the shortest average distance. As an example, the helical backbone native contacts for an $i$ to $i+4$ helix foldamer are shown in Figure 4. All instances of the optimal pairs are considered native contacts. Next, the distance tolerance $c_{t o l}$ for the trajectory scan is varied to maximize $Q_{1}-Q_{0}$. Further details regarding the native contacts optimization are given in the supporting information (Section S3). Over a wide range of both nonbonded and bonded force field parameters of helical foldamers, we have found that $c_{t o l}$ is generally approximately 1.3 (Figures S3-S4). For simplicity, we therefore set $c_{t o l}=1.3$ for all examples in Section 3 of this work to minimize the need for optimization of the native contact definitions. 


\subsection{Free energy, entropy, enthalpy of folding}

To compute the free energy change associated with molecular folding, the conformational states (i.e., "folded" and "unfolded") must first be given definitions by the researcher performing the calculation. If most conformational states are very clearly either folded or unfolded, i.e., in the case of a strongly cooperative foldamer with a single folded state, the free energy difference between them will be relatively insensitive to the precise definitions of the folded and unfolded states. However, if there is a not a clear division between the folded and unfolded states, different definitions of the conformational states may dramatically affect the results of the free energy calculation.

For the simple test systems considered in this study, we define the folded state as any structure which has a native contact fraction greater than $\frac{\left(Q_{0}+Q_{1}\right)}{2}$, using the optimized $Q$ vs. $T$ sigmoid fitting parameters. Conversely, any structure which has a native contact fraction less than or equal to $\frac{\left(Q_{0}+Q_{1}\right)}{2}$ is considered unfolded.

To compute free energy of folding as a function of temperature, given replica energy time series, replica trajectories, and corresponding native contact fractions, each frame in the trajectory is first classified as folded or unfolded. In general, classification criteria for $n$ conformational states can be used in cg_openmm. Next, MBAR is used to compute the probability expectations $\left\langle P_{i}\right\rangle$ at each temperature that a structure will be in each conformational state. The free energy change upon folding $\Delta G_{i j}(T)$, or generally upon any conformational state transition, can then be computed from the following relation:

$$
\Delta G_{i j}(T)=-k_{B} T \ln \left(\frac{\left\langle P_{i}\right\rangle}{\left\langle P_{j}\right\rangle}\right)
$$

We note that in the systems considered in this work, the volume of the simulation cell is not specified, and so $\Delta G$ is equivalent to the change in Helmholtz free energy $(\Delta A)$, and $\Delta H$ is equivalent to the change in internal energy $(\Delta U)$. Uncertainty in $\Delta G_{i j}(T)$ can be 
computed from the covariance matrix returned by pymbar, or by the bootstrapping scheme implemented in cg_openmm. From the free energy data as a function of temperature, entropy change of folding $(\Delta S)$ and enthalpy change of folding $(\Delta H)$ are computed by evaluating the derivative of a cubic spline fit to the $\Delta G$ vs $\mathrm{T}$ curve:

$$
\begin{aligned}
& \Delta S=-\left(\frac{\partial \Delta G}{\partial T}\right)_{P} \\
& \Delta H=\Delta G+T \Delta S
\end{aligned}
$$

Uncertainty in $\Delta S$ and $\Delta H$ is computed using the bootstrapping scheme described above. Example plots of free energy, entropy, and enthalpy of folding curves for a helix-forming homo-oligomer system are shown in Figure 5.
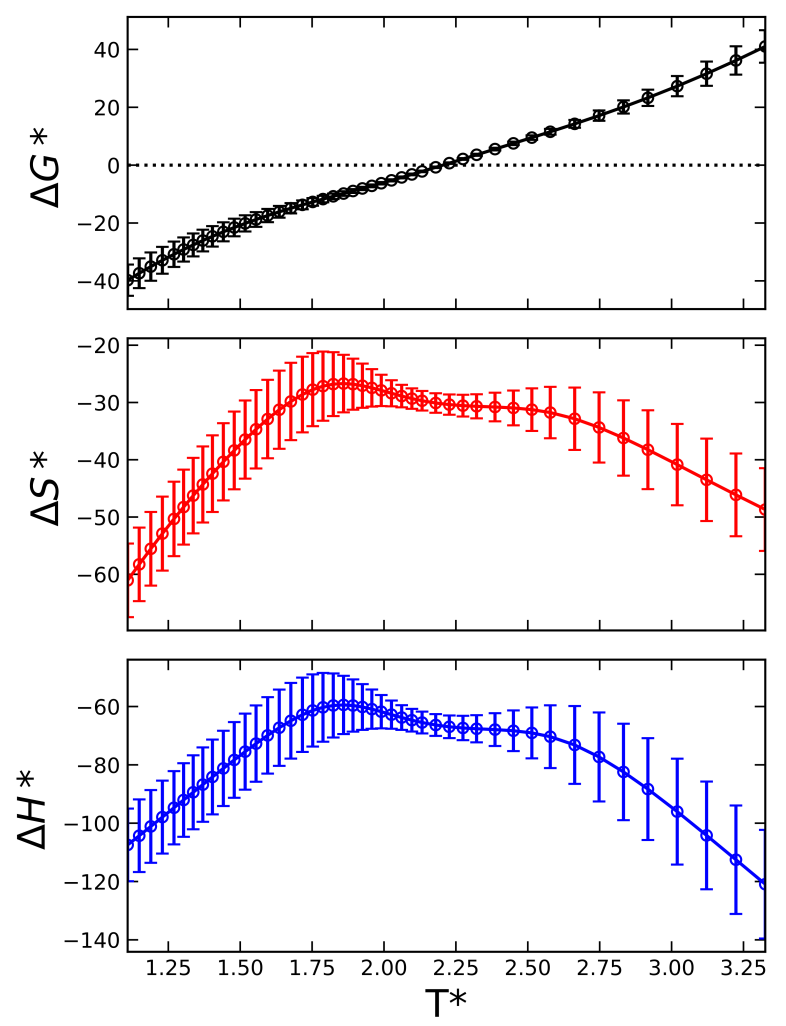

Figure 5: Example free energy of folding, entropy of folding, and enthalpy of folding curves for a helical foldamer system, with uncertainties estimated as the standard deviation from bootstrapping the native contact fraction expectations. All quantities are given in a reduced units system, in which $\varepsilon_{B}$ is the base energy unit. 


\subsection{Structural distributions}

The analyze_foldamers package is equipped with routines for computing bond length, bond-bending angle, and torsion angle distributions given a CGModel object and trajectory file(s). All types of bond, angle, and torsion particle sequences for an arbitrary heterooligomer are detected automatically from the topology in the CGModel, histogrammed, and saved to figure files. Of particular interest are the changes in bonded distributions over a set of replica exchange trajectories at different temperatures.

In addition to 1D histograms, analyze_foldamers contains a tool for plotting Ramachandran-like 2D histograms of backbone pseudotorsion angle and backbone bondbending angle, similar to the Ramachandran plots Tozzini and coworkers mapped from the atomistic $\phi / \psi$ angles to the coarse-grained $\alpha-\theta$ space. ${ }^{36} 2 \mathrm{D}$ histograms of any combination of two bonded parameters can also be generated in analyze_foldamers to investigate correlations amongst them throughout the folding transition.

\subsection{Workflows with Signac}

To streamline the workflow of running replica exchange simulations, post-processing to decorrelate time series data and write replica/state trajectory files, and performing structural and thermodynamic analysis operations, we have provided templates in cg_openmm for setting up Signac ${ }^{66}$ flow projects. Signac is a Python-based workflow manager, which allows the user to specify project operations and their dependencies using Python decorators. Ranges of force field and model parameters of interest are specified in a project's init.py script, and Signac manages the parameter scan directory structure, job data, and job submission on high-performance computing (HPC) clusters with minimal human input. Moreover, the Signac framework is highly scalable to very large parameter scans. 


\subsection{Helical modeling}

To obtain initial guesses for force field parameters of a minimalist 1-1 homo-oligomer model which folds cooperatively into a helix, we developed a simple helical modeling tool which optimizes helical radius $(r)$, pitch $(2 \pi c)$, and the spacing between Lennard-Jones particles $(\Delta t)$ placed along a helix, given the number of backbone particles $\left(n_{B}\right)$ and LennardJones $\sigma$ and $\varepsilon$ parameters. The spacing between backbone particles is assumed equal, such that the $\Delta t$ parameter fully defines the particle positions for a given radius and pitch. The objective function to minimize is the total energy of the particles placed along the helix, considering only the untruncated Lennard-Jones 12-6 function and no bonded terms nor exclusions. The helix is represented by the following parametric equations:

$$
\begin{gathered}
x=r \cos t \\
y=r \sin t \\
z=c t
\end{gathered}
$$

Sidechain particles are placed normal to the helix, at a distance from the corresponding backbone particle equal to the Euclidean distance between adjacent backbone particles at any given iteration (i.e., enforcing equal equilibrium bond lengths for B-B and B-S bonds). In this simplified model, sidechain particles impart no additional degrees of freedom to the system, but sidechain-sidechain and sidechain-backbone interactions are included.

The optimization is carried out using the differential_evolution solver in the SciPy package, ${ }^{56}$ which can more effectively navigate to the global energy minimum than gradientbased approaches. Using the positions of the energy-minimized helix, the values of all bonded

parameters for a given $\sigma$ and $\varepsilon$ can be estimated and tested in replica exchange MD simulations. The equilibrium bond distances, backbone bond-bending angle $\left(\theta_{B, o}\right)$, and backbone 
torsion angle $\left(\phi_{B, o}\right)$ in particular can be expected to closely match the values obtained from a full replica exchange MD simulation, provided that the corresponding force constants are adequately high. Given the constraint on the sidechain positions, however, parameters involving the sidechain are expected to require refinement. In future work, we aim to include more energy terms in the helical model, specifically bonded terms and the 1-2, 1-3 nonbonded exclusions, to provide even more accurate estimates of helix-stabilizing bonded parameters.

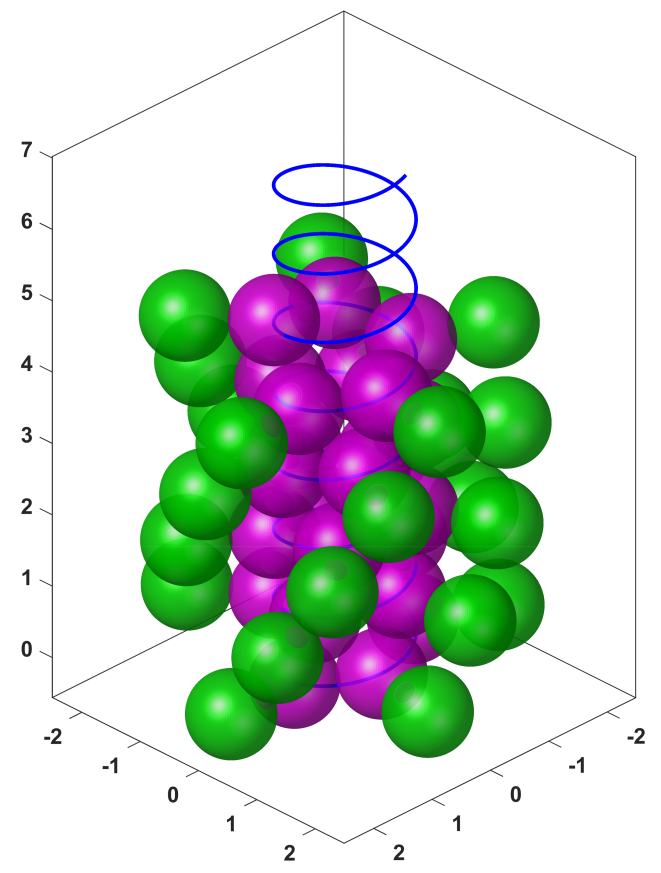

Figure 6: Illustration of the optimized backbone (magenta) and sidechain (green) particle positions spaced equally along the helical line (blue). All axes are in units of $\sigma_{B}$.

\section{Results and Discussion}

\subsection{Helical design}

To obtain a set of reasonable force field parameters forming the basis of various scans of nonbonded and bonded parameters, we first applied the helical modeling tool described 
above to a 1-1 model with 24 residues. The LJ 12-6 $\sigma$ parameter was set to a value of 1 for both sidechain and backbone particles, effectively defining the base unit of length in the system. The $\varepsilon$ parameters for each bead type were similarly set to 1 . In the case where all particles have the same LJ parameters, the optimized geometry of the helix is not affected by the value of $\varepsilon$. The helical parameters determined from the global energy minimization described in Section 2.7, with sidechain particles fixed normal to the helical line, are listed in Table 1, below, with distance units given in multiples of $\sigma$. For reference, in an $\alpha$-helix, $\theta_{B, o} \approx 92^{\circ}, \phi_{B, o} \approx 52^{\circ}$, and there are 3.6 residues per turn. ${ }^{29}$ The optimized helix model in this work has a wider backbone bond angle and smaller backbone torsion angle than the literature $\alpha$-helix, corresponding to a larger radius, smaller pitch, and more residues per turn.

Table 1: Results for helical optimization $(\sigma=1)$

\begin{tabular}{cc}
\hline Parameter & Value \\
\hline$r_{B-B}=r_{B-S}$ & 1.08 \\
$\theta_{B, o}$ (degrees) & 105.5 \\
$\phi_{B, o}$ (degrees) & 16.7 \\
radius & 0.86 \\
pitch & 0.96 \\
residues per turn & 4.73 \\
\hline
\end{tabular}

The geometry of the optimized helix provides estimates of equilibrium bond distance, bond-bending angles, and torsions angles. It does not, however, provide insight into the force constants or energetic parameters which stabilize the helix. Additionally, the constraint on the sidechain bead positions leads only to approximate positions, and this constraint should be removed in the MD simulations.

Before moving on to scanning the force field parameters to study stability and cooperativity of the designed helix, we make several simplifications to reduce the parameter space: 1) the backbone sigma parameter $\left(\sigma_{B}\right)$ is fixed at 1 (base distance unit), 2) the backbonebackbone and backbone-sidechain equilibrium bond lengths $\left(r_{B-B}, r_{B-S}\right)$ are fixed at a value of 1.08 determined from the positions of the optimized helix, 3) harmonic bonds are highly 
rigid with a dimensionless force constant of 506.25, and 4) all torsions involving sidechain beads are switched off for the sake of simplicity, such that the relevant 1-4 pairs interact only through the nonbonded potential. The masses of both particle types are fixed at 100 AMU, which only affects the dynamics, not the thermodynamics. Explicit sidechain torsions are expected to play an important role in facilitating folding, and will be revisited in future studies.

\subsection{Effect of varying sidechain nonbonded parameters}

We first examine the effect of the sidechain Lennard-Jones parameters on helix stability and cooperativity through a series of REMD simulations. These effects are assessed by analyzing the plots of heat capacity and average native contact fraction as functions of temperature. We will hereon refer to the plot of average value of native contact fraction versus temperature as simply "native contacts curve". A foldamer's melting point is a measure of stability, indicative of the level of resistance to thermal denaturation. The sigmoid width parameter $(d)$ of the native contacts curve and full-width half-maximum (FWHM) of the heat capacity curve are each measures of folding cooperativity. A more cooperative foldamer undergoes the folding transition over a narrower temperature range.

In a $2 \mathrm{D}$ grid of REMD simulations, we varied the sidechain energetic parameter $\varepsilon_{S}$ and sidechain size parameter $\sigma_{S}$. In each case, results were generated from 200 ns trajectories with constant entropy increase temperature spacing, as described above, following a preliminary $50 \mathrm{~ns}$ simulation with logarithmic temperature spacing. In addition to the fixed parameters specified in Section 3.1, the following bonded parameters were used, based on the geometry of the energy-minimized helix and reasonable estimates of force constants from preliminary simulations: $k_{a}=100 / \mathrm{rad}^{2}, k_{t}=3.33, \theta_{B, o}=105.5^{\circ}, \theta_{S, o}=127.5^{\circ}$, and $\phi_{B, o}=16.7^{\circ}$. This value of $\theta_{S, o}$ was chosen to satisfy the symmetry constraint $\theta_{B, o}+2 \theta_{S, o}=360^{\circ}$. 

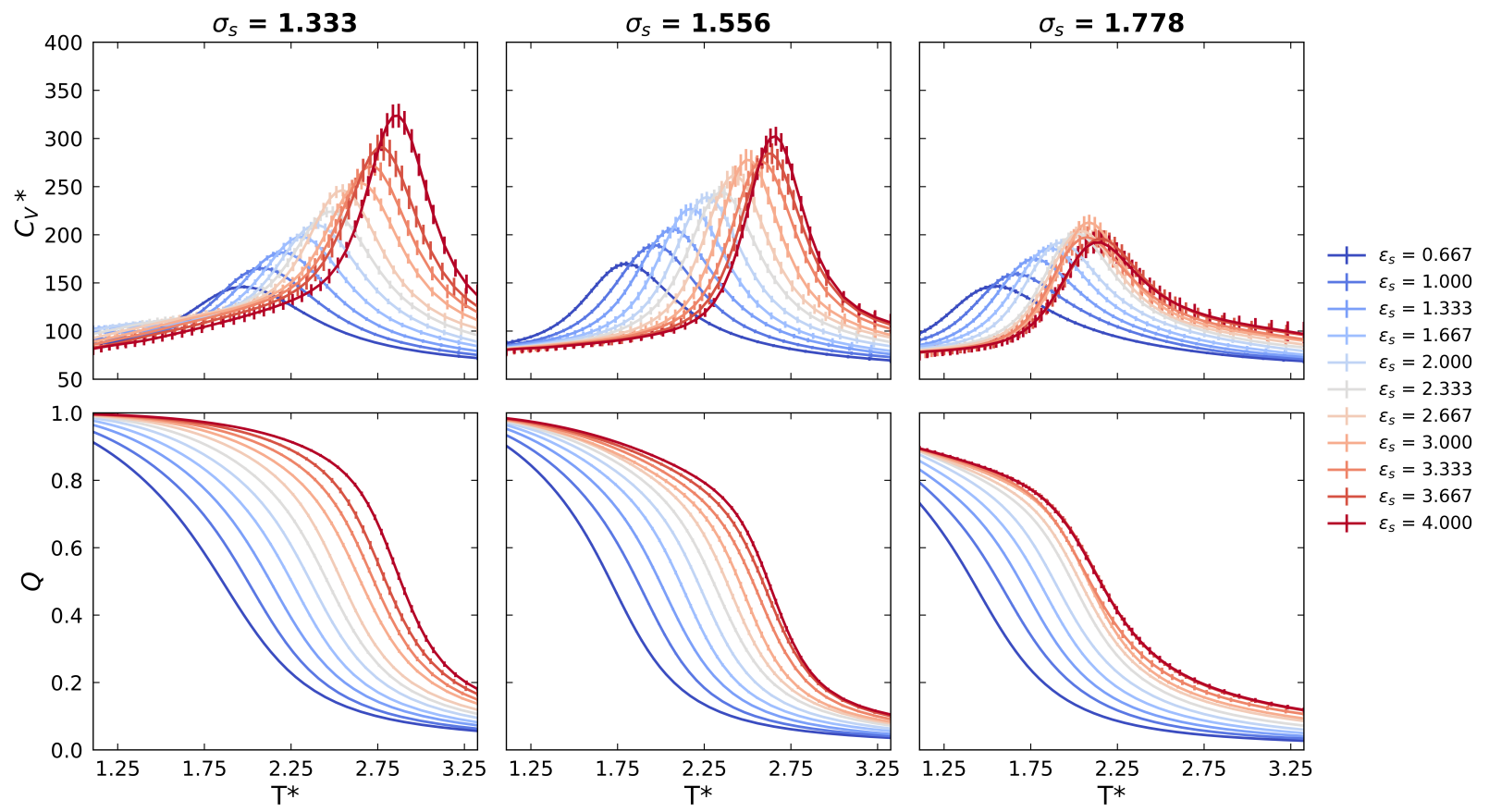

Figure 7: Top: the melting point (stability) computed from heat capacity increases with $\varepsilon_{S}$ and decreases with $\sigma_{S}$. Increasing $\varepsilon_{S}$ generally results in a larger $C_{V}$ peak height and narrower $C_{V}$ peak width (higher cooperativity). Bottom: native contact fraction expectation curves provide an alternative view of folding transition behavior using structural data rather than purely thermodynamic data. Trends in melting point (sigmoid center) and cooperativity (sigmoid characteristic width) are in excellent agreement with the heat capacity curves. Uncertainties represent the standard deviation from bootstrapping.

Tuning the sidechain Lennard-Jones parameters allows for fine control over the foldamer melting point (stability), height and width of the heat capacity curve (cooperativity), and even the helix geometry itself (pitch, radius). The heat capacity curves and corresponding native contacts curves for various combinations of $\sigma_{S}$ and $\varepsilon_{S}$ values are presented in Figure 7. Figure 8 shows 1D representations of key features of the heat capacity and native contacts curves in Figure 7. 

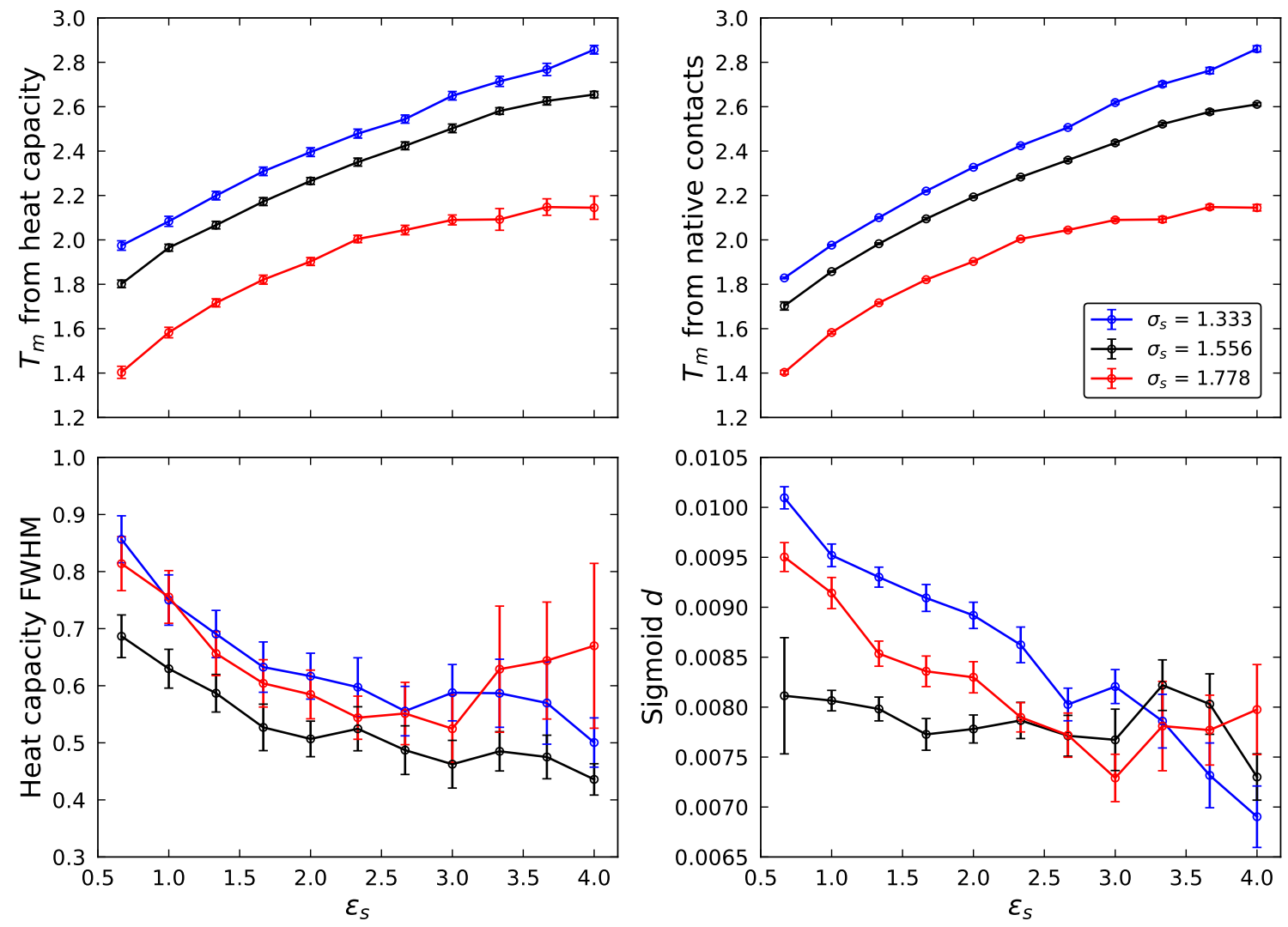

Figure 8: Helix stability increases with both $\varepsilon_{S}$ and $\sigma_{S}$ over the range of parameters studied, indicated by increases in melting point computed from heat capacity curves (top left) or native contacts curves (top right). Cooperativity increases roughly with increasing $\varepsilon_{S}$, as shown by decreases in the native contacts sigmoid width $(d)$ parameter (bottom right) and heat capacity full-width half-maximum (bottom left). Both metrics indicate that for low $\varepsilon_{S}$, using $\sigma_{S}=1.556$ results in the highest cooperativity. Uncertainties are estimated as the standard deviation from bootstrapping.

Helix stability (melting point) increases with sidechain interaction strength $\left(\varepsilon_{S}\right)$, and decreases with increasing sidechain size $\left(\sigma_{S}\right)$ due to stretching of the helical backbone. In particular, bulkier sidechain beads lead to higher pitch and slightly wider radius of the helical backbone. This helix stretching effect is most evident in the case of strong sidechain interactions (large $\varepsilon_{S}$ ). Figure 9 shows a representation of the ensemble of folded structures, determined from DBSCAN clustering, for three different values of $\sigma_{S}$ and with $\varepsilon_{S}=4$. Further details on the DBSCAN clustering protocol and cluster statistics are provided in the supporting information (Section S4). Detailed changes in structural distributions, including 
backbone angle, sidechain angle, and backbone torsion for these three parameter sets are also shown in the supporting information (Figure S7). Given that melting point decreases with increasing $\sigma_{S}$ for all $\varepsilon_{S}$ studied (Figure 8), the bulkiness of the sidechain beads has a destabilizing effect. Since this destabilization is largely due to stretching of the helix, adjusting the equilibrium backbone bond-bending angle and torsion angle could counter this loss of stability for systems with bulky sidechains.

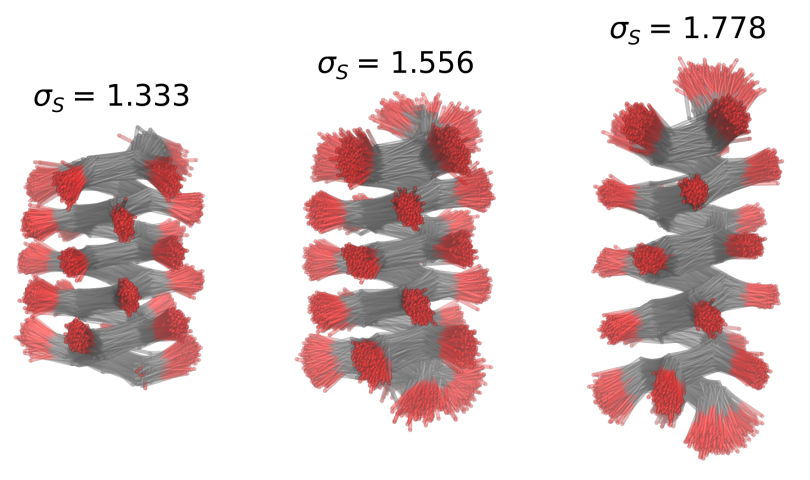

Figure 9: Representative samples from the folded clusters for $\varepsilon_{S}=4$ and varying $\sigma_{S}$ illustrate changes in helical pitch with $\sigma_{S}$. Structures are aligned with respect to the cluster medoids.

It is also evident that there is an upper limit to $\varepsilon_{S}$ for achieving a stable helix. For $\sigma_{S}=1.778$, interaction strengths above $\varepsilon_{S}=3.0$ no longer increase the melting point. At higher $\varepsilon_{S}$, the strong sidechain-sidechain and sidechain-backbone interactions begin to distort/destabilize the helix. We also considered $\varepsilon_{S}$ up to 5.0, and observed this plateauing of the melting point for smaller $\sigma_{S}$ as well. Heat capacity and native contacts curves for these additional values of $\varepsilon_{S}$ are provided in the supporting information (Figure S11).

In addition to shifting the melting point, the sidechain energetic parameter $\varepsilon_{S}$ significantly increases the height and sharpness of the heat capacity peak, and therefore the cooperativity of the folding transition. While the sigmoid width $(d)$ is roughly equal (within the bootstrapping uncertainty of 1 standard deviation) for all $\sigma_{S}$ considered at high $\varepsilon_{S}$, at low $\varepsilon_{S}$ it is clear that the intermediate $\sigma_{S}$ parameter $\left(1.556 \sigma_{B}\right)$ results in the strongest cooperativity. In contrast, the smallest $\sigma_{B}$ leads to the weakest cooperativity at low $\varepsilon_{S}$. This is expected since the range of the sidechain interactions is small for low $\sigma_{B}$. The heat capacity 
FWHM results mirror these trends in cooperativity, with the moderate $\sigma_{S}$ exhibiting the sharpest heat capacity peak for all $\varepsilon_{S}$.

\subsection{Effect of varying equilibrium backbone angles and torsions}

Next, we explore the stability of the designed helix to changes in the backbone equilibrium bond-bending angle $\left(\theta_{B, o}\right)$ and backbone equilibrium torsion angle $\left(\phi_{B, o}\right)$ through a series of REMD simulations. Along with the fixed parameters specified in Section 3.1, the following force field parameters were used: $\sigma_{S}=1.556, \varepsilon_{S}=3.33, k_{a}=100 / \mathrm{rad}^{2}$, and $k_{t}=3.33$. We also applied the symmetry constraint that $2 \theta_{S, o}+\theta_{B, o}=360^{\circ}$, a simplification that we study further in the subsequent section.

While cooperativity measures changed only marginally for the different $\theta_{B, o}$ and $\phi_{B, o}$ considered, stability of the designed helix varied significantly with $\theta_{B, o}$. The effect of the bond-bending angle on stability dominates the effect of torsion angle for the parameter ranges considered here. Figure 10 shows the heat capacity and native contact fraction curves for varying equilibrium bond-bending angle values, with a backbone torsion equilibrium angle of $16^{\circ}$, and Figure 11 shows the melting points and characteristic widths from both heat capacity and native contacts curves for the complete $2 \mathrm{D}$ scan of $\theta_{B, o}$ and $\phi_{B, o}$. As may be anticipated, the melting point is highest for the foldamer with angle parameters closest to that in the designed helix (see Table 1). 

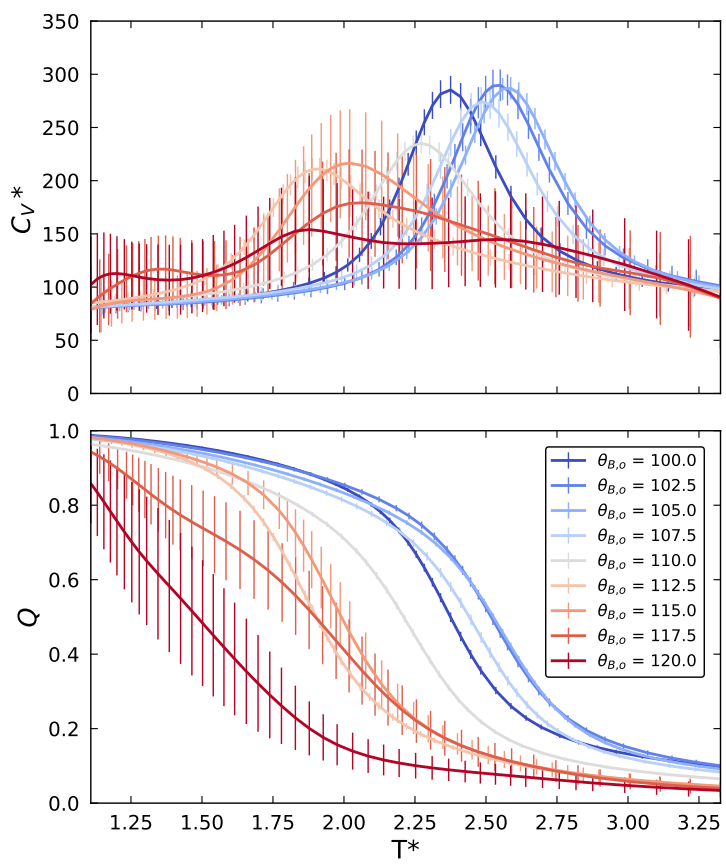

Figure 10: The melting point (stability) of the helix foldamer can be modulated by varying the equilibrium backbone bond-bending angle $\left(\theta_{B, o}\right)$. With sufficient increases to $\theta_{B, o}$ the helix is destabilized, as seen by broad heat capacity curves and non-sigmoidal native contacts curves. Curves shown are for $\phi_{B, o}=16^{\circ}$, and uncertainties are estimated as the standard deviation from bootstrapping. 

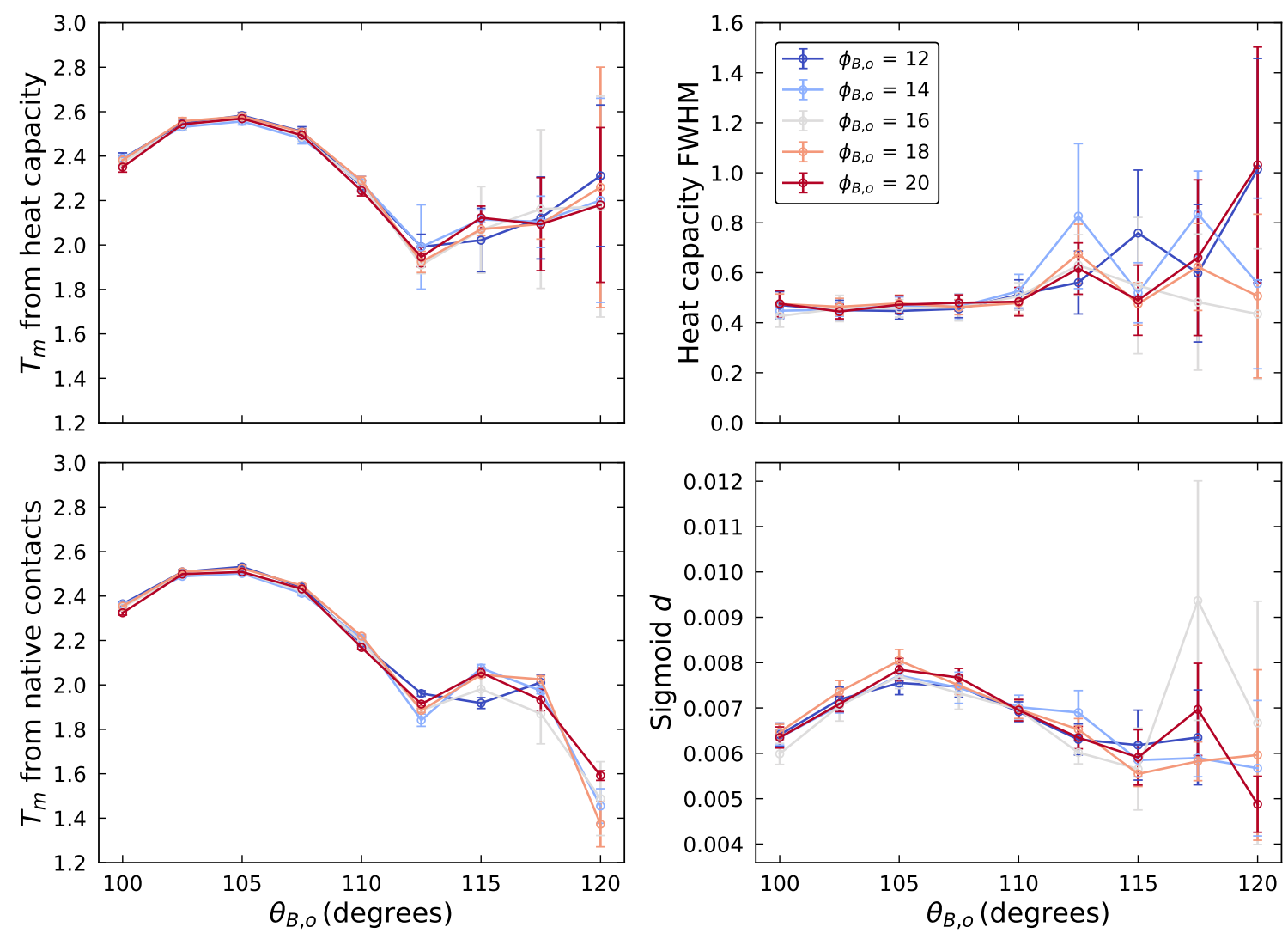

Figure 11: Melting point of the foldamer computed from the heat capacity curve (top left) and native contacts curve (bottom left) reveal that the $(i$ to $i+5)$ helix is less stable than the $(i$ to $i+4)$ helix. Heat capacity full-width half-maximum (top right) and sigmoid width parameter (bottom right) indicate that cooperativity is not significantly different for the $(i$ to $i+4)$ and $(i$ to $i+5)$ helices. In addition, the effect of perturbing the backbone torsion angle on melting point and characteristic width of the transition region is negligible over the parameter range tested. For the native contact sigmoid parameters, the data point for $\phi_{B, o}=12^{\circ}, \theta_{B, o}=120^{\circ}$, has been omitted due to the non-sigmoidal behavior of the native contacts curve. All uncertainties are estimated as the standard deviation from bootstrapping.

Moreover, we found that complete structural transitions can occur with very small bonded parameter changes. A clear structural transition between two stable helices occurs upon increasing the backbone bond-bending angle by a small amount from $110^{\circ}$ to $112.5^{\circ}$, as shown by the shift in the backbone bond-bending angle and backbone torsion angle distributions with the change in bond-bending angle (Figure 12). In this structural transition, the optimal native contact pairs (defined by the smallest average distance in the native structure) change 
from $(i$ to $i+4)$ spacing for $110^{\circ}$, to $(i$ to $i+5)$ spacing for $112.5^{\circ}$. The melting point data shows that the $(i$ to $i+4)$ helix is more stable for the set of force field parameters considered here. Knowledge of the molecular parameters which lead to a helix-to-helix transition provides valuable guidance on how to design foldamers which toggle between the two helices in response to a stimulus, for example.
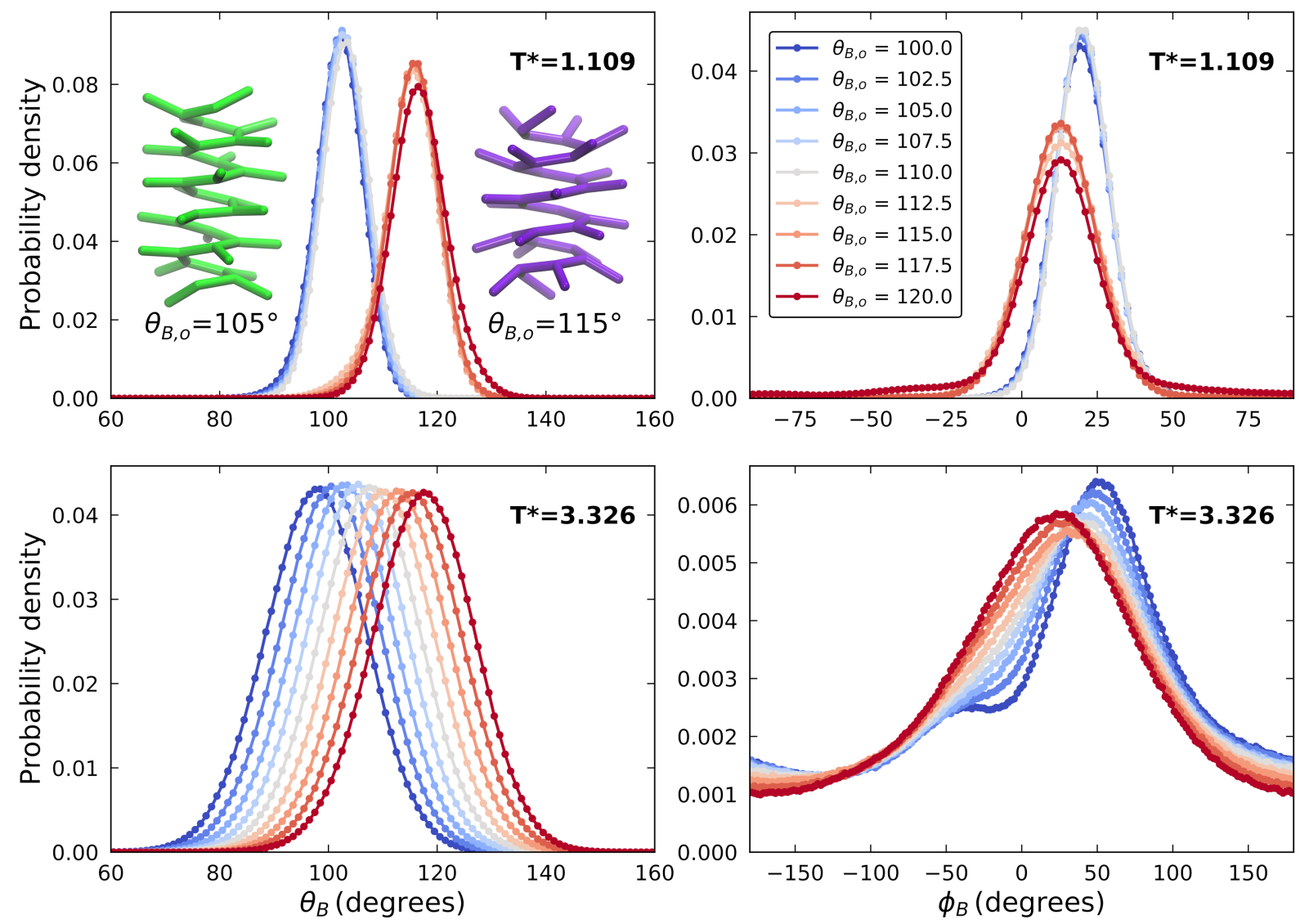

Figure 12: Increasing the equilibrium value of the backbone bond-bending angle causes a sharp transition over a range of just $2.5^{\circ}$ from a $(i$ to $i+4)$ helix (green) to a wider ( $i$ to $i+5$ ) helix (purple), as evidenced from shifts in the backbone bond-bending angle distributions (left) and backbone torsion angle distributions (right). All distributions shown are for $\phi_{B, o}=16^{\circ}$. Structures are medoids from the cluster with the lowest RMSD amongst members, after a final energy minimization.

\subsection{Effect of varying equilibrium sidechain angle}


In this section we explore moving the sidechain angle equilibrium value $\left(\theta_{S, o}\right)$ away from the value enforced by symmetry $\left(360^{\circ}-\theta_{B, o}\right) / 2$ in Sections 3.2 and 3.3. This symmetry constraint was enforced in the helix designed in section 3.1, in which the sidechain beads were fixed normal to the helical backbone. In addition to the fixed parameters specified in Section 3.1, the following force field parameters were used: $\sigma_{S}=1.556, \varepsilon_{S}=3.33$, $k_{a}=100 / \mathrm{rad}^{2}, k_{t}=3.33, \theta_{B, o}=105.5^{\circ}, \phi_{B, o}=16.7^{\circ}$.
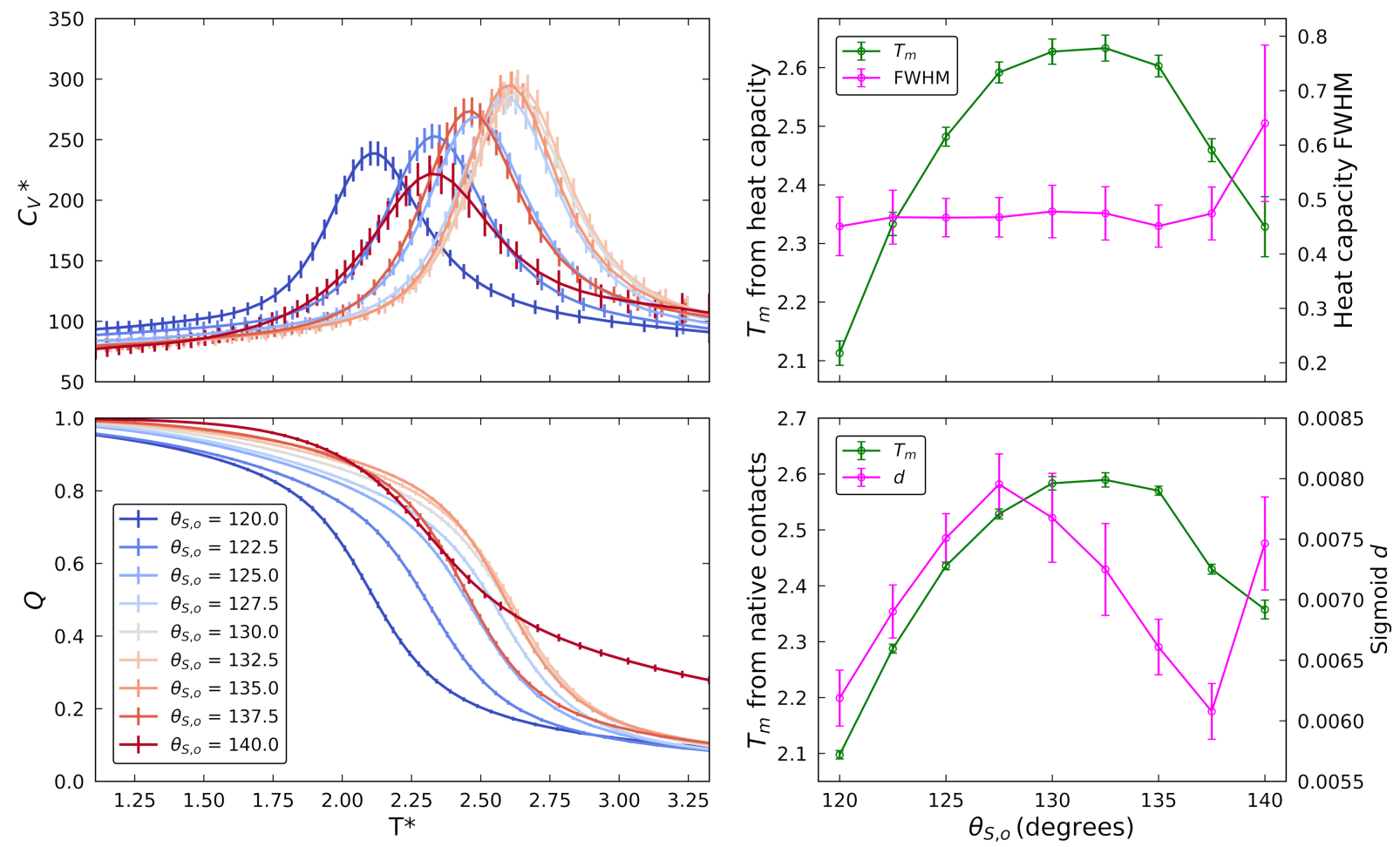

Figure 13: Sidechain angle equilibrium values near the expected value from symmetry $\left(127.5^{\circ}\right)$ lead to the most stable helices, as indicated by a maximum in melting points calculated from both the heat capacity and native contacts curves. The width of heat capacity curves (upper right) is constant for all $\theta_{S, o}$ until the helix becomes distorted at $\theta_{S, o}=140^{\circ}$. The sigmoid width of the native contacts curves (lower right), in contrast, is largest for $\theta_{S, o}=127.5$. For all $\theta_{S, o}$, the backbone equilibrium angle is set at $105.5^{\circ}$, and an angle force constant of $100 / \mathrm{rad}^{2}$ is applied to both angle types. All uncertainties are estimated as the standard deviation from bootstrapping.

From the heat capacity and native contacts curves computed from REMD simulations with varying $\theta_{S, o}$ (Figure 13), it is apparent that even small perturbations to the equilibrium value of the sidechain angle have a large impact on the melting point and height of the 
heat capacity peak. Cooperativity, measured by the full-width half-maximum of the heat capacity curve, was unaffected by $\theta_{S, o}$ except for the largest sidechain angle considered. The sidechain and backbone angle distributions for different $\theta_{S, o}$ (Figure 14) show that increasing $\theta_{S, o}$ from 137.5 to 140.0 degrees severely distorts the helix. The backbone structure of the helix is minimally disrupted by changes to the sidechain angle until that destabilization occurs. Interestingly, $\theta_{S, o}$ near the value from the symmetry constraint $\left(127.5^{\circ}\right)$ exhibits a maximum in melting point. The symmetry constrained value of $\theta_{S, o}$ provides sidechain beads with the most space in a helical conformation. Even at high temperature, changes in $\theta_{S}$ cause a shift in the distribution of $\theta_{B}$ angles, suggesting a correlation between $\theta_{S}$ and $\theta_{B}$. These findings confirm that constraining the sidechain angles at $\left(360^{\circ}-\theta_{B, o}\right) / 2$ is a valid assumption for maximal stability for these sets of force field parameters used. Significant deviations from the symmetry value in either direction destabilize the helix. 

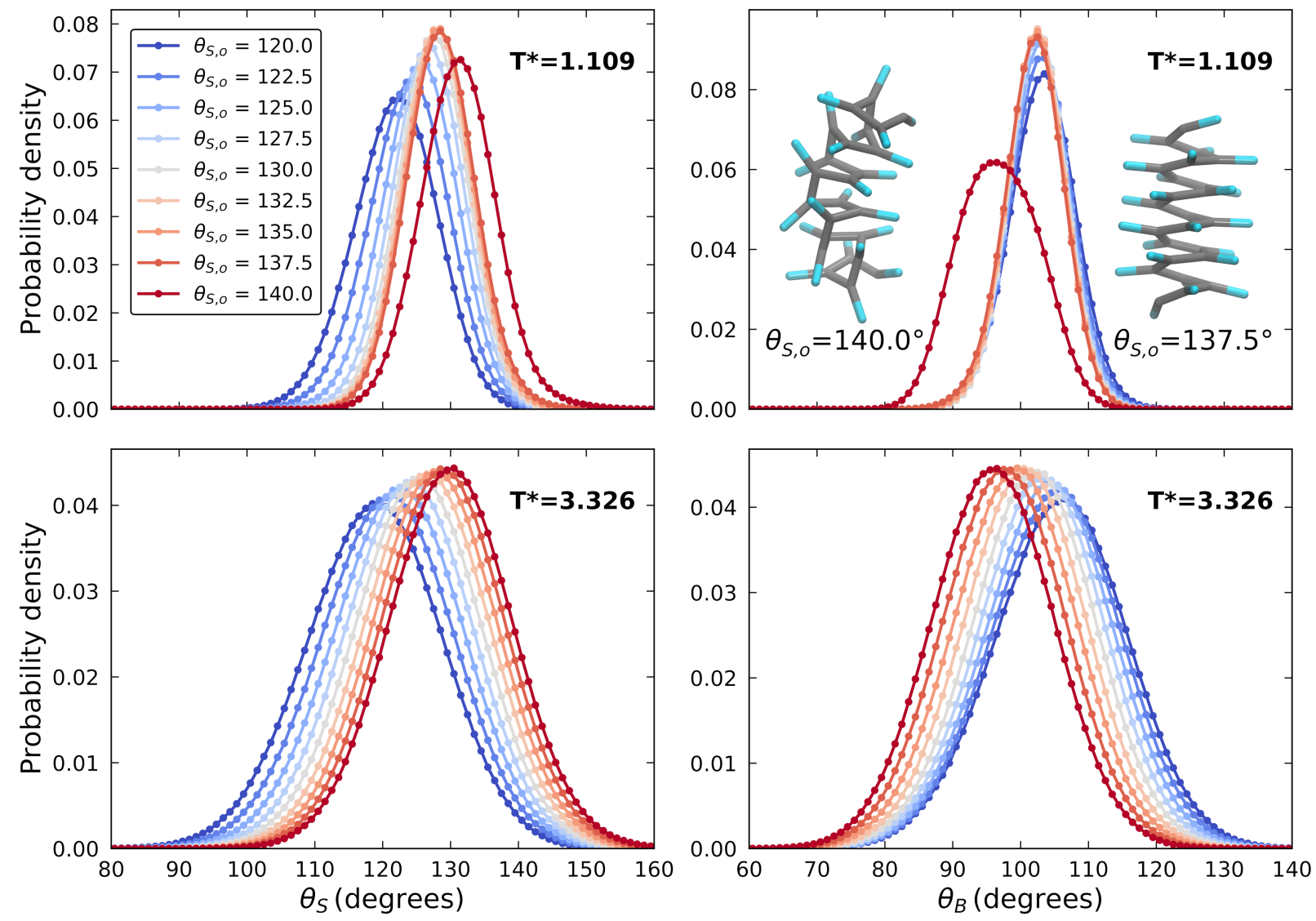

Figure 14: Bond-bending angle distributions of the sidechain (left) and backbone (right) show that increasing the sidechain angle equilibrium value from $137.5^{\circ}$ to $140.0^{\circ}$ destabilizes the designed helix. Higher maxima in the $\theta_{S}$ distribution also signify higher stability of the helix. Given that the backbone equilibrium angle is set at $105.5^{\circ}$ in all cases, angle distributions in the unfolded state at high temperature (bottom) indicate a correlation between $\theta_{B}$ and $\theta_{S}$. Structures shown are medoids from the cluster with the lowest RMSD amongst members, after a final energy minimization.

\subsection{Effect of varying angle and torsion stiffness}

Next, we investigated how the backbone angle stiffness and backbone torsion stiffness affect helix stability and folding cooperativity. We ran a 2D grid of REMD simulations varying the force constants $k_{a}$ and $k_{t}$, with all other nonbonded and bonded parameters kept constant. Specifically, we set $\sigma_{S}=1.556, \varepsilon_{S}=3.33, \theta_{B, o}=105.5^{\circ}, \theta_{S, o}=127.5^{\circ}$, and $\phi_{B, o}=16.7^{\circ}$. As before, explicit sidechain torsion potentials are set to zero, with 1-4 
interactions governed only by the standard 1-4 nonbonded interactions.

We found that backbone torsion stiffness strongly affects cooperativity, whereas angle stiffness has a much weaker influence on cooperativity. In addition, stability (melting point) increases with both angle and torsion stiffness. The heat capacity curves and corresponding native contact fraction curves are given in Figure 15 for an angle force constant of 100/rad ${ }^{2}$ and a series of backbone torsion force constants ranging from 0 (no torsion forces) to 4.667. The sigmoid width parameter of the native contacts curves, full-width half maximum of the heat capacity curve, and the melting point computed from both the heat capacity and native contacts curves, are also plotted. For each of the angle force constants considered, torsion force constants of 0.667 and lower do not sufficiently stabilize the helical backbone, leading to broad heat capacity curves and ill-defined native contacts (omitted from the native contact fraction plots). Due to the slower kinetics of folding for low torsion force constant, replica exchange simulations for $k_{t}=1.333$ and lower were run for $400 \mathrm{~ns}$ instead of $200 \mathrm{~ns}$, with the last 100 ns used as the production period. Of the torsion force constants tested, values of 1.333-2.000 lead to the most cooperative transition, characterized by the smallest sigmoid width and heat capacity full-width half-maximum parameters. Upon increasing the torsion force constant from 2.0, the sigmoid width increases and the melting point increases, highlighting the tradeoff between high cooperativity and high stability. These trends in characteristic width and melting point hold true for all values of $k_{a}$ considered. 

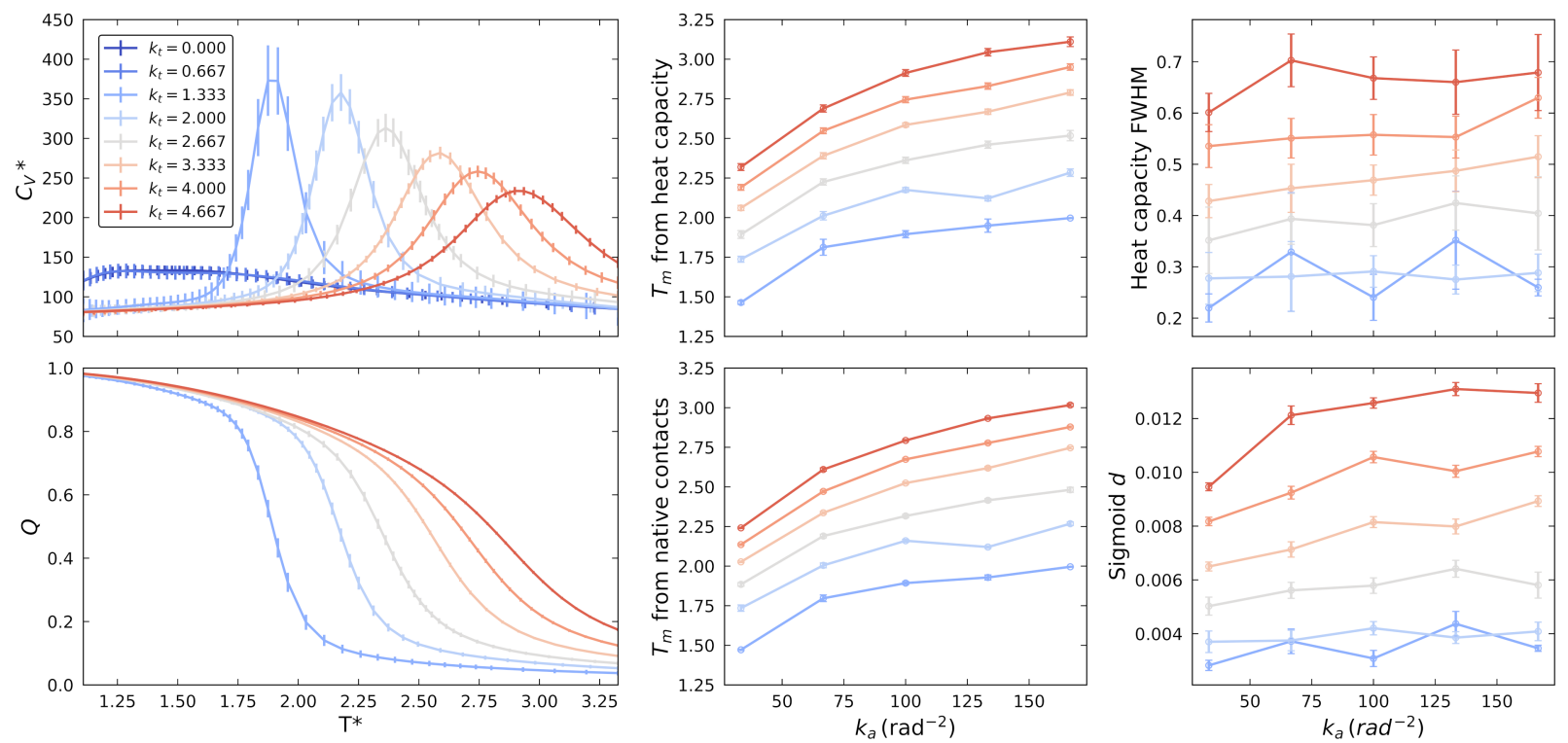

Figure 15: For a given backbone angle stiffness, cooperativity is maximal for a torsion force constant of 1.333-2.000, as indicated by the smallest sigmoid width and heat capacity fullwidth half-maximum parameters (right). Cooperativity metrics are relatively unaffected by the angle force constant over the range studied. Heat capacity and native contacts curves (left) are shown for an angle force constant of $100 / \mathrm{rad}^{2}$. For $k_{t}=0.667$ and below, the helix is not sufficiently stable to be able to produce a native contacts curve. For $k_{t}=1.333$ and above, the melting point (stability) increases with both angle and torsion stiffness (middle). All uncertainties are estimated using standard deviations from bootstrapping.

\subsection{Effect of varying chain length}

As mentioned, the choice of 24 residue chain length used throughout this work is a fairly arbitrary choice. However, there are lower and upper bounds for forming stable helicesoligomers that are too short may not have enough native contacts to stabilize the helical backbone, and oligomers that are too long may entropically prefer to form more complex secondary or even tertiary structures involving hairpins. In this section, we repeated the heat capacity and native contacts analysis on replica exchange simulations of oligomers with varying chain length, ranging from 6 residues to 36 residues.

In addition to the fixed parameters specified in Section 3.1, the following force field parameters were used: $\sigma_{S}=1.556, \varepsilon_{S}=3.33, k_{a}=100 / \mathrm{rad}^{2}, k_{t}=2.0, \theta_{B, o}=105.5^{\circ}$, 
$\phi_{B, o}=16.7^{\circ}$. We chose the torsion force constant of 2.0 based on the results of the previous section, in which the heat capacity peak height and width were found to be optimal. As the kinetics of folding for $k_{t}=1.333$ are expected to be slower than for $k_{t}=2.0$, we chose the stiffer of the two torsion force constants.

Due to the larger time scales associated with the folding of the longer chains, especially with the relatively weak torsion force constant used, each constant entropy increase replica exchange simulation was run for $400 \mathrm{~ns}$, following a $50 \mathrm{~ns}$ preliminary simulation with log temperature spacing. The first $300 \mathrm{~ns}$ were discarded as the equilibration period in all cases. As with all previous simulations, 12 replicas were used with the same upper and lower temperature bounds.

For the chain lengths where helices are stable, cooperativity increases with the number of residues, and stability is highest for 18-24 residues. The heat capacity curves (on a perresidue basis) and corresponding native contacts curves are plotted in Figure 16. The medoid structures taken from the cluster with the lowest intra-cluster RMSD, with subsequent energy minimization, are also shown for each chain length considered. For the case of 6 residues, there are too few native contacts along the backbone to stabilize the helix, and no peak in heat capacity is observed over the temperature range used. For 12 through 30 residues, the expected helix structure emerges. For 12 residues, the heat capacity peak and transition region in the native contacts curve are very broad, indicating weak cooperativity and/or stability. Increasing the number of residues from 18 to 24 to 30 , the width of the heat capacity curve decreases, the melting point decreases, and the height of the heat capacity curve increases. This corresponds to increasing cooperativity and decreasing stability. Upon increasing chain length to 36 residues, additional structure emerges - a strand of $\sim 10$ residues in an extended conformation forms adjacent to the helix containing the remaining $\sim 26$ residues. Despite using the helical backbone native contacts scheme for the 36 residue system, the melting point and characteristic width of the native contacts curve parallels that of the heat capacity curve. However, the native contact fraction in the folded state is artificially 
low, since the strand-helix contacts were not considered.
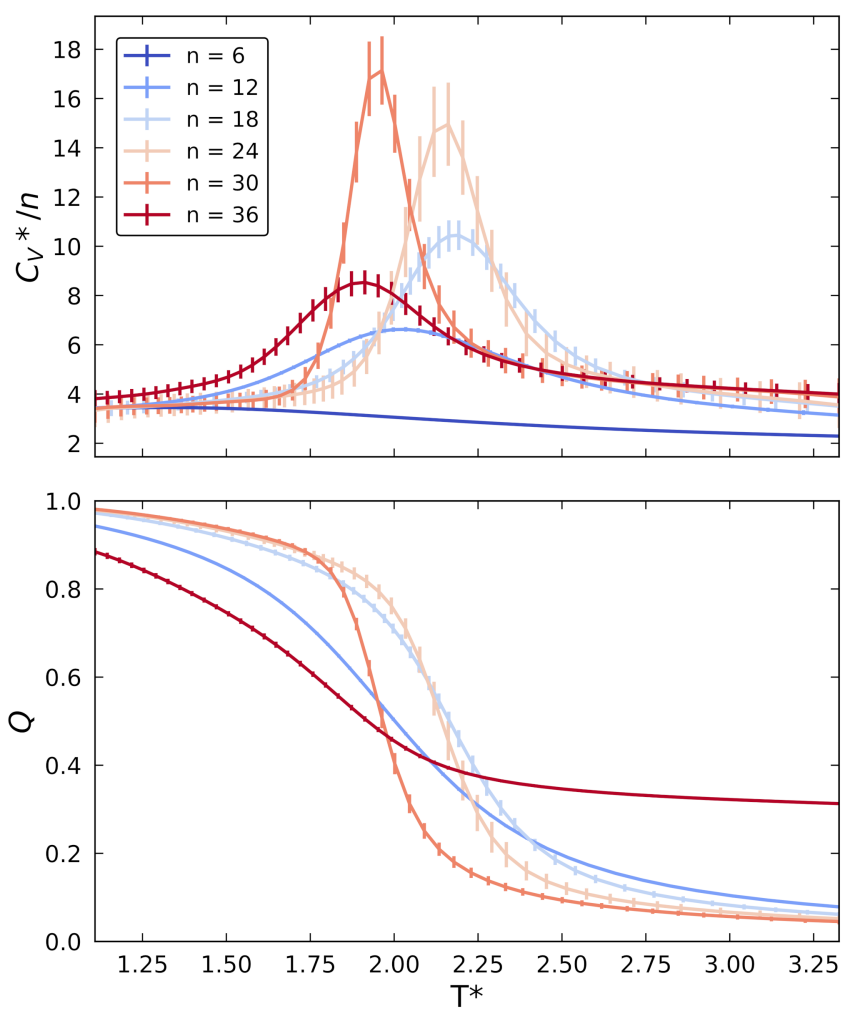
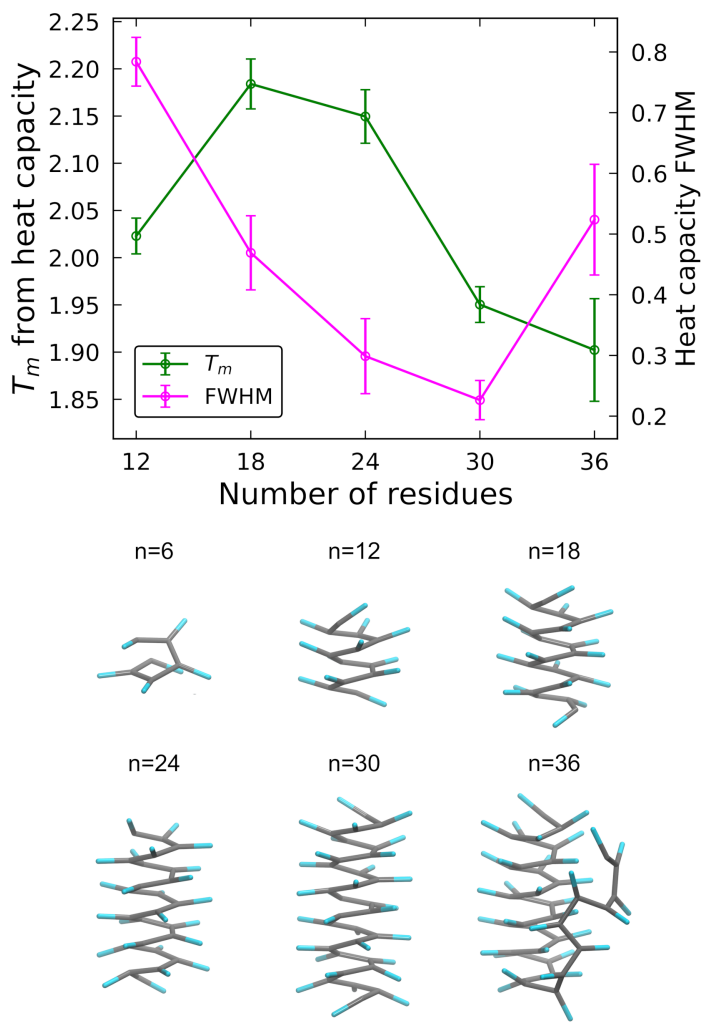

Figure 16: With increasing chain length, the width of the heat capacity peak at half maximum, a rough measure of cooperativity of folding, decreases until tertiary structure emerges at 36 residues. The melting point computed from the heat capacity curve is maximal for 18-24 residues. Similar behavior is indicated by the position and steepness of the transition in the native contacts curve (lower left). Shorter chains have fewer native contacts to provide stability, while longer chains begin to favor formation of a strand adjacent to the helix. Energy-minimized medoids for varying numbers of residues in a helical foldamer, taken from the cluster with lowest intra-cluster RMSD in each case, are shown. All uncertainties are estimated using the standard deviation from bootstrapping.

\section{Conclusions}

In this work, we introduced the Python package cg_openmm which facilitates coarsegrained modeling and thermodynamic analysis of potential foldamer molecules, and analyze_foldamers, a companion Python package which performs structural analysis, in- 
cluding determination of native structure from RMSD clustering and calculation of bonded parameter distributions. Given a coarse-grained model, temperature replica exchange molecular dynamics simulations are performed using OpenMM, and MBAR reweighting is applied to the decorrelated replica energies to accurately calculate heat capacity, native contact fraction expectation, and free energy of folding as functions of temperature. Heat capacity and native contacts curves can then be analyzed to study the stability and cooperativity of a potential foldamer system.

To demonstrate the capabilities of cg_openmm, we examined a 24 residue homo-oligomer system which we designed to fold into a helix. Guided by initial estimates for bonded parameters derived from a helical modeling tool included in cg_openmm, we systematically varied sets of force field parameters and investigated the effects on cooperativity and stability of the designed helix. Specifically, we varied the sidechain size and energetic Lennard-Jones parameters (Section 3.2), backbone angle and backbone torsion equilibrium values (Section 3.3), sidechain angle equilibrium value with fixed backbone equilibrium angle (Section 3.4), and backbone angle stiffness and backbone torsion stiffness (Section 3.5). In addition, we varied the number of residues using a set of force field parameters found to produce highly cooperative folding in the 24 residue model (Section 3.6).

In all sets of experiments, we found that even small changes to force field parameters can profoundly affect the stability, cooperativity, and even the geometry of the helix. For example, the helical pitch could be tuned in a continuous manner by varying $\sigma_{S}$. By varying the backbone angle equilibrium value by just 2.5 degrees, we observed a transition between two helices with different pitch, radius, and backbone native contact sequences. This type of insight hints at design pathways for switchable materials. In many cases we also determined the parameter bounds for achieving a stable helix. Features which prevented cooperative folding into helices included too weak of a backbone torsion force constant, too strong sidechain interactions $\left(\varepsilon_{S}\right)$, too many residues, and angle values that deviated too far from those in the original helix model. 
It is important to state that these findings are valid only in the context of the other force field parameters. Cooperativity of folding and stability of any secondary structural element are complex functions of the entire set of force field parameters, including some not considered here explicitly such as sidechain torsion potentials. To further improve cooperativity in homo-oligomer coarse-grained models, it will likely be necessary to move beyond ad hoc force field parameter scans, and instead use gradient or global optimization techniques.

While we observed significant cooperativity of folding for the simple homo-oligomer models considered here, the potential of homo-oligomer foldamers is hampered by a limited design space. To achieve highly cooperative folding transitions and discover novel secondary structures, it will be necessary to consider heteropolymers with multiple side chain and/or backbone types.

Heat capacity and native contact fraction expectation curves offer complementary perspectives on folding behavior - the former based solely on thermodynamic data, and the latter on both structural and thermodynamic data. Generally, we see excellent agreement between melting points computed from heat capacity curves and those computed from the sigmoid fit to the native contacts curve. However, some limitations with the current method of describing native contacts are apparent when comparing the two cooperativity metrics, sigmoid width parameter and heat capacity FWHM. For example, when varying the sidechain equilibrium angle $\left(\theta_{S, o}\right)$, the heat capacity curve shows no change in FWHM from $120^{\circ}$ to $137.5^{\circ}$, whereas the native contacts sigmoid width exhibits a maximum at $127.5^{\circ}$ (Figure 13 ). A definition of native contacts that is a continuous function of pairwise distances, rather than a discrete function that depends on a cutoff and a distance tolerance factor, would be of great interest for improving the accuracy of native contacts curves and thereby the free energy of folding and other quantities derived from it.

Foldamer parameters discovered using cg_openmm can guide the design of atomistic foldamers in the laboratory. Reverse-mapping (also called "backmapping"), the process of inserting atomistic detail into coarse-grained models, has been used successfully to achieve 
equilibrated macromolecular configurations that would be difficult to attain with only allatom simulations. ${ }^{67,68}$ Since our coarse-grained models are not chemically specific, the challenge is then to determine which (synthetically accessible) chemistries best describe the geometry and interaction potentials present in the coarse-grained system. Then, all-atom simulations can be run to verify and fine-tune the folding characteristics.

The cg_openmm and analyze_foldamers packages and documentation are available under MIT license through GitHub at https://github.com/shirtsgroup/cg_openmm and https://github.com/shirtsgroup/analyze_foldamers, respectively.

\section{Author Contributions}

G.A.M., M.R.S, and T.L.F. conceptualized the project; G.A.M. originally built the software, with additional significant contributions from C.C.W. and M.R.S.; The methodology was designed by G.A.M., C.C.W., T.L.F. and M.R.S.; C.C.W. primarily wrote the original draft with contributions from M.R.S and G.A.M.; M.R.S., T.L.F., and G.A.M. edited and reviewed the manuscript; All experiments were carried out by C.C.W; Experiments were analyzed and validated by C.C.W. and M.R.S.; M.R.S. supervised the project and obtained the resources.

\section{Acknowledgement}

T.L.F. acknowledges support from a GAANN fellowship. This material is based upon work supported by the U.S. Department of Energy, Office of Science, Office of Basic Energy Sciences, Materials Sciences and Engineering (MSE) Division, under Award Number DESC0018651. This work utilized computational resources from the University of Colorado Boulder Research Computing Group, which is supported by the National Science Foundation (awards ACI-1532235 and ACI-1532236), the University of Colorado Boulder, and Colorado State University. This work also used the Extreme Science and Engineering Discovery Environment (XSEDE), which is supported by National Science Foundation grant number 
ACI-1548562. Specifically, it used the Bridges system, which is supported by NSF award number ACI-1445606, and the Bridges-2 system, which is supported by NSF ACI-1928147, both at the Pittsburgh Supercomputing Center (PSC). Finally, the authors acknowledge the openmmtools team for useful discussion and help regarding the implementation of the replica exchange simulations, especially John Chodera, Andrea Rizzi, Ana Silviera, and Josh Fass.

\section{Supporting Information Available}

The following files are available free of charge.

- supporting_info.pdf: Comparison of replica transition matrices for logarithmic and optimized temperature spacing, description of physical validation analysis, description of native contacts determination, description of clustering protocol, supplementary structural distributions for the sidechain LJ parameter scan simulations, results for additional sidechain LJ parameters considered, and results for repeated trials of selected simulations with independent starting configurations.

\section{References}

(1) Gopalakrishnan, R.; Frolov, A. I.; Knerr, L.; Drury, W. J.; Valeur, E. Therapeutic Potential of Foldamers: From Chemical Biology Tools To Drug Candidates? Journal of Medicinal Chemistry 2016, 59, 9599-9621.

(2) Choi, S.; Isaacs, A.; Clements, D.; Liu, D.; Kim, H.; Scott, R. W.; Winkler, J. D.; DeGrado, W. F. De novo design and in vivo activity of conformationally restrained antimicrobial arylamide foldamers. Proceedings of the National Academy of Sciences of the United States of America 2009, 106, 6968-6973.

(3) Tew, G. N.; Scott, R. W.; Klein, M. L.; Degrado, W. F. De novo design of antimicrobial 
polymers, foldamers, and small molecules: From discovery to practical applications. Accounts of Chemical Research 2010, 43, 30-39.

(4) Müller, M. M.; Windsor, M. A.; Pomerantz, W. C.; Gellman, S. H.; Hilvert, D. A rationally designed aldolase foldamer. Angewandte Chemie - International Edition 2009, $48,922-925$.

(5) Maayan, G.; Ward, M. D.; Kirshenbaum, K. Folded biomimetic oligomers for enantioselective catalysis. Proceedings of the National Academy of Sciences of the United States of America 2009, 106, 13679-13684.

(6) Prince, R. B.; Barnes, S. A.; Moore, J. S. Foldamer-based molecular recognition. Journal of the American Chemical Society 2000, 122, 2758-2762.

(7) Lee, B. C.; Chu, T. K.; Dill, K. A.; Zuckermann, R. N. Biomimetic nanostructures: Creating a high-affinity zinc-binding site in a folded nonbiological polymer. Journal of the American Chemical Society 2008, 130, 8847-8855.

(8) Nam, K. T.; Shelby, S. A.; Choi, P. H.; Marciel, A. B.; Chen, R.; Tan, L.; Chu, T. K.; Mesch, R. A.; Lee, B. C.; Connolly, M. D.; Kisielowski, C.; Zuckermann, R. N. Freefloating ultrathin two-dimensional crystals from sequence-specific peptoid polymers. Nature Materials 2010, 9, 454-460.

(9) Gellman, S. H. Foldamers: A Manifesto. Accounts of Chemical Research 1998, 31, $173-180$.

(10) Hill, D. J.; Mio, M. J.; Prince, R. B.; Hughes, T. S.; Moore, J. S. A field guide to foldamers. Chemical Reviews 2001, 101, 3893-4011.

(11) Huc, I. Aromatic Oligoamide Foldamers. European Journal of Organic Chemistry 2004, $17-29$. 
(12) Cheng, R. P. Beyond de novo protein design - De novo design of non-natural folded oligomers. Current Opinion in Structural Biology 2004, 14, 512-520.

(13) Goodman, C. M.; Choi, S.; Shandler, S.; DeGrado, W. F. Foldamers as versatile frameworks for the design and evolution of function. Nature Chemical Biology 2007, 3, $252-262$.

(14) Horne, W. S.; Gellman, S. H. Foldamers with heterogeneous backbones. Accounts of Chemical Research 2008, 41, 1399-1408.

(15) Saraogi, I.; Hamilton, A. D. Recent advances in the development of aryl-based foldamers. Chemical Society Reviews 2009, 38, 1726-1743.

(16) Pilsl, L. K.; Reiser, O. $\alpha / \beta$-Peptide foldamers: State of the art. Amino Acids 2011, 41, 709-718.

(17) Guichard, G.; Huc, I. Synthetic foldamers. Chemical Communications 2011, 47, 59335941.

(18) Zhang, D. W.; Zhao, X.; Hou, J. L.; Li, Z. T. Aromatic amide foldamers: Structures, properties, and functions. Chemical Reviews 2012, 112, 5271-5316.

(19) Martinek, T. A.; Fülöp, F. Peptidic foldamers: Ramping up diversity. Chemical Society Reviews 2012, 41, 687-702.

(20) Bernardi, R. C.; Melo, M. C.; Schulten, K. Enhanced sampling techniques in molecular dynamics simulations of biological systems. Biochimica et Biophysica Acta - General Subjects 2015, 1850, 872-877.

(21) Wang, A. H.; Zhang, Z. C.; Li, G. H. Advances in enhanced sampling molecular dynamics simulations for biomolecules. Chinese Journal of Chemical Physics 2019, 32, $277-286$. 
(22) Shirts, M. R.; Chodera, J. D. Statistically optimal analysis of samples from multiple equilibrium states. Journal of Chemical Physics 2008, 129, 124105.

(23) Naden, L. N.; Shirts, M. R. Rapid Computation of Thermodynamic Properties over Multidimensional Nonbonded Parameter Spaces Using Adaptive Multistate Reweighting. Journal of Chemical Theory and Computation 2016, 12, 1806-1823.

(24) Brini, E.; Simmerling, C.; Dill, K. Protein storytelling through physics. Science 2020, 370.

(25) Alford, R. F. et al. The Rosetta All-Atom Energy Function for Macromolecular Modeling and Design. Journal of Chemical Theory and Computation 2017, 13, 3031-3048.

(26) Torrisi, M.; Pollastri, G.; Le, Q. Deep learning methods in protein structure prediction. Computational and Structural Biotechnology Journal 2020, 18, 1301-1310.

(27) Lutz, J.-F., Ed. Sequence-controlled polymers; Wiley-VCH: Weinheim, 2018.

(28) Nerenberg, P. S.; Head-Gordon, T. New developments in force fields for biomolecular simulations. Current Opinion in Structural Biology 2018, 49, 129-138.

(29) Tozzini, V. Quarterly Reviews of Biophysics; 2010; Vol. 43; pp 333-371.

(30) Kmiecik, S.; Gront, D.; Kolinski, M.; Wieteska, L.; Dawid, A. E.; Kolinski, A. CoarseGrained Protein Models and Their Applications. Chemical Reviews 2016, 116, 78987936.

(31) Hills, R. D.; Brooks, C. L. Insights from coarse-grained Gō models for protein folding and dynamics. International Journal of Molecular Sciences 2009, 10, 889-905.

(32) Reith, D.; Pütz, M.; Müller-Plathe, F. Deriving effective mesoscale potentials from atomistic simulations. Journal of Computational Chemistry 2003, 24, 1624-36. 
(33) Izvekov, S.; Voth, G. A. A multiscale coarse-graining method for biomolecular systems. The Journal of Physical Chemistry B 2005, 109, 2469-73.

(34) Shell, M. S. In Advances in Chemical Physics, 1st ed.; Rice, S. A., Dinner, A. R., Eds.; John Wiley \& Sons, Inc., 2016; Vol. 161; pp 395-441.

(35) Wang, J.; Chmiela, S.; Müller, K.-R.; Noé, F.; Clementi, C. Ensemble Learning of Coarse-Grained Molecular Dynamics Force Fields with a Kernel Approach. Journal of Chemical Physics 2020, 152, 194106.

(36) Tozzini, V.; Rocchia, W.; McCammon, J. A. Mapping all-atom models onto one-bead coarse-grained models: General properties and applications to a minimal polypeptide model. Journal of Chemical Theory and Computation 2006, 2, 667-673.

(37) Zhou, J.; Thorpe, I. F.; Izvekov, S.; Voth, G. A. Coarse-grained peptide modeling using a systematic multiscale approach. Biophysical Journal 2007, 92, 4289-4303.

(38) Eastman, P.; Swails, J.; Chodera, J. D.; McGibbon, R. T.; Zhao, Y.; Beauchamp, K. A.; Wang, L. P.; Simmonett, A. C.; Harrigan, M. P.; Stern, C. D.; Wiewiora, R. P.; Brooks, B. R.; Pande, V. S. OpenMM 7: Rapid development of high performance algorithms for molecular dynamics. PLoS Computational Biology 2017, 13, 1-17.

(39) Periole, X.; Marrink, S. J. Methods in Molecular Biology; 2013; Vol. 924; Chapter 20, pp 533-565.

(40) Sugita, Y.; Okamoto, Y. Replica-exchange molecular dynamics method for protein folding. Chemical Physics Letters 1999, 314, 141-151.

(41) Periole, X.; Mark, A. E. Convergence and sampling efficiency in replica exchange simulations of peptide folding in explicit solvent. Journal of Chemical Physics 2007, 126, 014903. 
(42) Qi, R.; Wei, G.; Ma, B.; Nussinov, R. Replica exchange molecular dynamics: A practical application protocol with solutions to common problems and a peptide aggregation and self-assembly example. Methods in Molecular Biology 2018, 1777, 101-119.

(43) Ahn, S. H.; Grate, J. W. Foldamer Architectures of Triazine-Based Sequence-Defined Polymers Investigated with Molecular Dynamics Simulations and Enhanced Sampling Methods. Journal of Physical Chemistry B 2019, 123, 9364-9377.

(44) Abraham, M. J.; Gready, J. E. Ensuring mixing efficiency of replica-exchange molecular dynamics simulations. Journal of Chemical Theory and Computation 2008, 4, 11191128.

(45) Sindhikara, D.; Meng, Y.; Roitberg, A. E. Exchange frequency in replica exchange molecular dynamics. Journal of Chemical Physics 2008, 128, 024103.

(46) Kofke, D. A. On the acceptance probability of replica-exchange Monte Carlo trials. Journal of Chemical Physics 2002, 117, 6911-6914.

(47) Rathore, N.; Chopra, M.; De Pablo, J. J. Optimal allocation of replicas in parallel tempering simulations. Journal of Chemical Physics 2005, 122, 024111.

(48) Sabo, D.; Meuwly, M.; Freeman, D. L.; Doll, J. D. A constant entropy increase model for the selection of parallel tempering ensembles. Journal of Chemical Physics 2008, 128, 174109.

(49) Trebst, S.; Troyer, M.; Hansmann, U. H. Optimized parallel tempering simulations of proteins. Journal of Chemical Physics 2006, 124, 174903.

(50) Predescu, C.; Predescu, M.; Ciobanu, C. V. On the Efficiency of Exchange in Parallel Tempering Monte Carlo Simulations. Journal of Physical Chemistry B 2005, 109, 41894196. 
(51) Chodera, J. D.; Shirts, M. R. Replica exchange and expanded ensemble simulations as Gibbs sampling: Simple improvements for enhanced mixing. Journal of Chemical Physics 2011, 135, 194110.

(52) Friedrichs, M. S.; Eastman, P.; Vaidyanathan, V.; Houston, M.; Legrand, S.; Beberg, A. L.; Ensign, D. L.; Bruns, C. M.; Pande, V. S. Accelerating Molecular Dynamic Simulation on Graphics Processing Units. Journal of Computational Chemistry 2012, $30,864-872$.

(53) Eastman, P.; Pande, V. S. Efficient Nonbonded Interactions for Molecular Dynamics on a Graphics Processing Unit. Journal of Computational Chemistry 2010, 31, 1268-1272.

(54) Eastman, P.; Pande, V. S. OpenMM: A Hardware-Independent Framework for Molecular Simulations. Computing in Science and Engineering 2010, 12, 34-39.

(55) Lelièvre, T.; Rousset, M.; Stoltz, G. Free Energy Computations: A Mathematical Perspective; Imperial College Press: London, 2010.

(56) Virtanen, P. et al. SciPy 1.0: fundamental algorithms for scientific computing in Python. Nature Methods 2020, 17, 261-272.

(57) Shirts, M. R. Simple quantitative tests to validate sampling from thermodynamic ensembles. Journal of Chemical Theory and Computation 2013, 9, 909-926.

(58) Merz, P. T.; Shirts, M. R. Testing for physical validity in molecular simulations. PLoS ONE 2018, 13, 1-22.

(59) Efron, B.; Tibshirani, R. J. An introduction to the Bootstrap; Chapman \& Hall/CRC: Boca Raton, FL, 1993.

(60) Peng, J. H.; Wang, W.; Yu, Y. Q.; Gu, H. L.; Huang, X. Clustering algorithms to analyze molecular dynamics simulation trajectories for complex chemical and biological systems. Chinese Journal of Chemical Physics 2018, 31, 404-420. 
(61) McGibbon, R. T.; Beauchamp, K. A.; Harrigan, M. P.; Klein, C.; Swails, J. M.; Hernández, C. X.; Schwantes, C. R.; Wang, L. P.; Lane, T. J.; Pande, V. S. MDTraj: A Modern Open Library for the Analysis of Molecular Dynamics Trajectories. Biophysical Journal 2015, 109, 1528-1532.

(62) Schubert, E.; Sander, J.; Ester, M.; Kriegel, H.-P.; Xu, X. DBSCAN revisited, revisited: Why and how you should (still) use DBSCAN. ACM Transactions on Database Systems 2017, 42.

(63) Ester, M.; Kriegel, H.-P.; Sander, J.; Xu, X. A Density-Based Algorithm for Discovering Clusters in Large Spatial Databases with Noise. 2nd International Conference on Knowledge Discovery and Data Mining. Portland, Oregon, 1996; pp 226-231.

(64) Pedregosa, F.; Varoquaux, G.; Gramfort, A. Scikit-learn: Machine Learning in Python. Journal of Machine Learning Research 2011, 12, 2825-2830.

(65) Wang, K.; Yang, Y.; Chodera, J. D.; Shirts, M. R. Identifying ligand binding sites and poses using GPU-accelerated Hamiltonian replica exchange molecular dynamics. Journal of Computational Aided Molecular Design 2013, 27, 989-1007.

(66) Adorf, C. S.; Dodd, P. M.; Ramasubramani, V.; Glotzer, S. C. Simple data and workflow management with the signac framework. Computational Materials Science 2018, 146 , $220-229$.

(67) Wassenaar, T. A.; Pluhackova, K.; Böckmann, R. A.; Marrink, S. J.; Tieleman, D. P. Going backward: A flexible geometric approach to reverse transformation from coarse grained to atomistic models. Journal of Chemical Theory and Computation 2014, 10, 676-690.

(68) Peng, J.; Yuan, C.; Ma, R.; Zhang, Z. Backmapping from Multiresolution CoarseGrained Models to Atomic Structures of Large Biomolecules by Restrained Molecular 
Dynamics Simulations Using Bayesian Inference. Journal of Chemical Theory and Computation 2019, 15, 3344-3353. 
Graphical TOC Entry

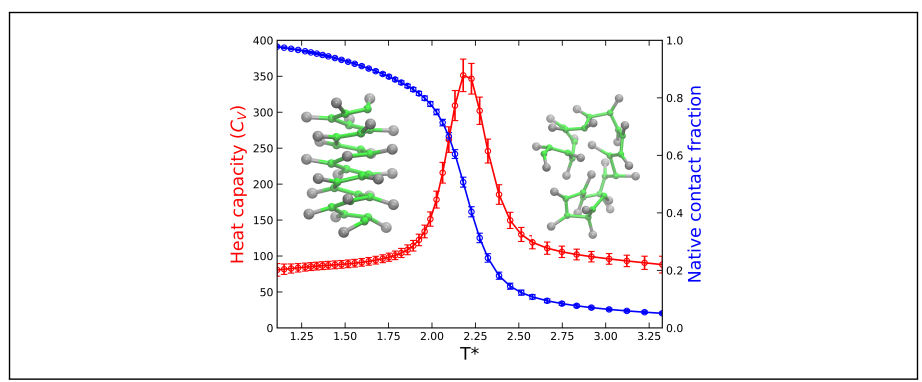

